\title{
Experimental study of the wake behind a surface-piercing cylinder for a clean and contaminated free surface
}

\author{
By AMY WARNCKE LANG $\dagger$ AND MORTEZA GHARIB \\ Graduate Aeronautical Laboratories, California Institute of Technology, \\ Pasadena, CA 91125, USA
}

(Received 31 March 1998 and in revised form 5 August 1999)

This experimental investigation into the nature of free-surface flows was to study the effects of surfactants on the wake of a surface-piercing cylinder. A better understanding of the process of vorticity generation and conversion at a free surface due to the absence or presence of surfactants has been gained. Surfactants, or surface contaminants, have the tendency to reduce the surface tension proportionally to the respective concentration at the free surface. Thus when surfactant concentration varies across a free surface, surface tension gradients occur and this results in shear stresses, thus altering the boundary condition at the free surface. A low Reynolds number wake behind a surface-piercing cylinder was chosen as the field of study, using digital particle image velocimetry (DPIV) to map the velocity and vorticity field for three orthogonal cross-sections of the flow. Reynolds numbers ranged from 350 to 460 and the Froude number was kept below 1.0. In addition, a new technique was used to simultaneously map the free surface deformation. Shadowgraph imaging of the free surface was also used to gain a better understanding of the flow. It was found that, depending on the surface condition, the connection of the shedding vortex filaments in the wake of the cylinder was greatly altered with the propensity for surface tension gradients to redirect the vorticity near the free surface to that of the surface-parallel component. This result has an impact on the understanding of turbulent flows in the vicinity of a free surface with varying surface conditions.

\section{Introduction}

In recent years, the role that surfactants can play with respect to the dynamics of free-surface flows has been realized to be of significant importance in understanding the behaviour of vorticity and turbulence in general at a free surface. The damping effect that surfactants can have on waves or other perturbing deformations at a free surface is well known. The effect of surface contamination on damping eddies at a free surface was studied by Davies (1966). The interaction of vortex pairs with a contaminated and clean parallel free surface has been studied experimentally and computationally by Bernal et al. (1989), Tryggvason et al. (1992), Tsai \& Yue (1995), and Hirsa \& Willmarth (1994). They observed opposite-sign vorticity being generated at the free surface in the contaminated case, as opposed to a rebounding of the pair in the clean case. Gharib \& Weigand (1996) looked at the connection of an inclined

$\dagger$ Present address: Department of Aerospace and Mechanical Engineering, Parks College of Engineering and Aviation, Saint Louis University, St. Louis, MO 63103, USA. 


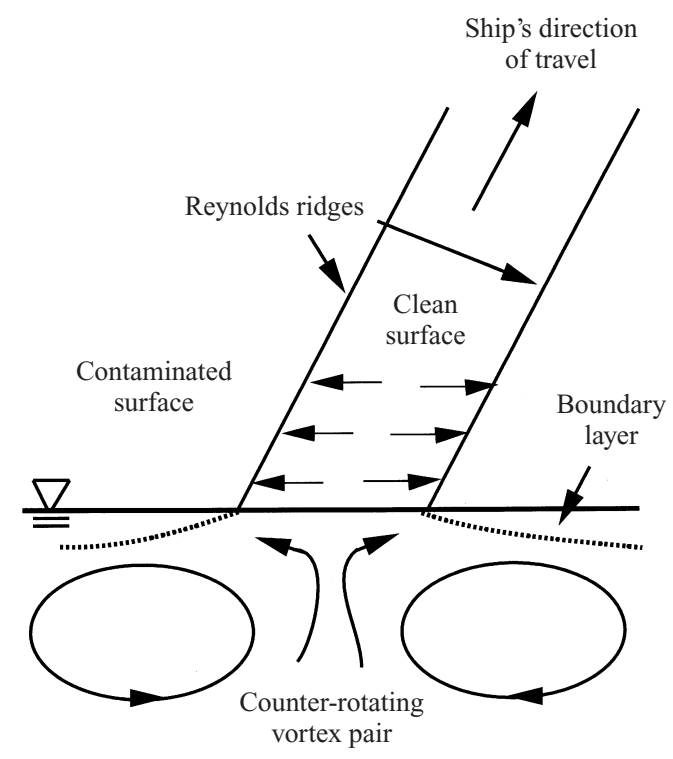

FIGURE 1. Schematic of the flow field in the far wake of a ship.

vortex ring to a free surface. In the clean case they observed the formation of two separate half rings, while in the contaminated case only one half ring was formed as the connection process was altered by the surface contamination. Finally, Willert \& Gharib (1997) observed the interaction of a modulated vortex pair with a free surface. They also observed differences with the connection process depending on the cleanliness of the free surface, with the greater tendency for the vorticity to connect normally in the clean case.

The study of coherent vortex structures near a free surface is of particular interest in their relevance to ship wakes. Surfactants, certainly of varying composition and thus varying rheological properties, abound at the ocean surface and thus an understanding of their impact on flows near a free surface is of significant importance. While the Reynolds numbers of these wakes based on ship length are usually in excess of $10^{8}$, insight into them can be gained from more simple examples of vortex dynamics in the vicinity of a free surface. Figure 1 shows a schematic of the averaged far-field largescale flow in ship wakes that is currently accepted by most to occur from observation and experimentation. A counter-rotating vortex pair in an area very close to the free surface is generated due to the interaction of the boundary layer on the hull of the ship with the ocean surface (Lyden, Lyzenga \& Shuchman 1986 and Willert 1992). The diverging surface current is also an aspect found when studying a jet parallel to the free surface (Walker 1997). In addition, asymmetry in the wake is observed in the real case due to several reasons such as propeller rotation (Meadows et al. 1994; Marmorino \& Trump 1996). Thus the simple flow model in figure 1 with the diverging surface current at the wake centreline is due to both the hull/free-surface interaction and net propulsion of the ship moving through the ocean. The ocean surface is contaminated, and even more so in the case of the ship wake. Surfactants, by their nature, tend to stay at a free surface due to both the existence of hydrophobic and hydrophilic ends on opposite sides of the molecule and the degree of solubility of the surfactant. Thus fresh water brought to the surface by the vortex pair, and 
any bubbles generated by the breaking waves near the ship, will carry additional surfactants to the ocean surface.

In addition, at the ocean surface, which can be treated as an unbounded domain, there is a minimum tangential velocity at the free surface which is required to compress the surfactant layer and break it apart to form the relatively clean region shown in figure 1. This minimum velocity is essentially the speed at which a surfactant layer will spread over the surface, and is dependent upon the elasticity (and thus kind of surfactant), concentration, and thickness of the film near its leading edge. The demarcation between the clean and contaminated surfaces is known as a Reynolds ridge. Warncke, Gharib \& Roesgen (1996) were the first to measure the boundary layer that forms beneath the ridge (see figure 1). They also showed that the vorticity flux, in this case that forms the boundary layer, is due to the sharp deceleration of the free-surface velocity across the ridge. Thus the surface tension gradient across the ridge provides the boundary condition at the free surface to which the flow must dynamically conform by decelerating and creating a shear stress at the surface.

In the case presented here, the simple vortex/free-surface interaction studied is that of convecting, normal, vortex filaments shed from a cylinder. The experiment was performed using digital particle image velocimetry (DPIV) to study the three orthogonal cross-sections of the flow. In addition, two surface imaging techniques were used to look at the free-surface deformation field. These results give increased knowledge of the effects of surfactants on the near-surface vorticity distribution in a simple bluff body wake.

\section{Theory}

\subsection{Free-surface boundary conditions}

Gharib \& Weigand (1996) describe the boundary conditions for the case of a flat free surface depending on the adjoining medium. In this case, which by definition is then a shear-free surface, they show that the only component of vorticity allowed to exist at the surface is the surface-normal component. In the case of a solid surface (stationary), they show that due to the no-slip condition at the wall only surfaceparallel vorticity can exist at the surface. Thus, in the case of a clean surface vortex filaments, which cannot terminate within the fluid due to the kinematic laws of vortex motion, will have the tendency to connect normally at the free surface. However, in the case of the solid boundary, disconnected filaments cannot attach to the surface normally but will tend to connect with nearby vorticity of the same rotation.

For a flat contaminated surface the presence of surfactants can result in surface tension gradients. As a result the boundary condition is written as

$$
\tau_{r s}=\tau_{\text {AIR }}+\frac{\partial \sigma}{\partial s}+\left(\kappa^{s}+\mu^{s}\right) \frac{\partial^{2} u_{s}}{\partial s^{2}}
$$

where an equation presented by Gharib \& Weigand (1996) is used along with one from Edwards, Brenner \& Wasan (1991) to derive the last term, and $s$ is the surface component. Here the dilitational $\left(\kappa^{s}\right)$ and shear $\left(\mu^{s}\right)$ viscosities of the surfactant film are included to account for their rheological effects at the free surface. The shear due to air, the adjoining medium, is assumed negligible and thus the surface tension and acceleration of the film must be balanced by the shear stress within the fluid. As a result, neither the shear-free nor the no-slip conditions apply and all components of vorticity may be present at a flat contaminated free surface. 


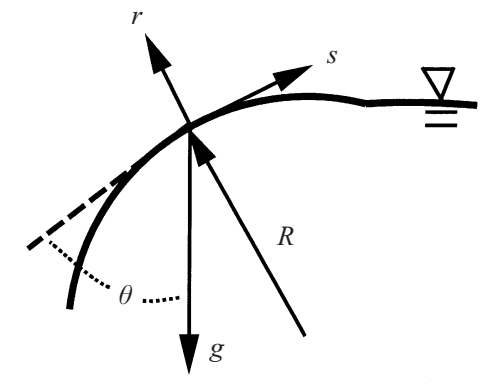

FIGURE 2. Local curvilinear coordinate system.

\subsection{Vorticity and vorticity flux}

Using the curvilinear coordinate system defined in figure 2, the shear stress condition at the free surface, and the equation given by Gharib \& Weigand (1996), the surfaceparallel vorticity (out of the page, or $z$-direction) is defined as

$$
\omega_{z}=-\frac{\tau_{\mathrm{AIR}}}{\mu}-\frac{1}{\mu}\left(\frac{\partial \sigma}{\partial s}+\left(\kappa^{s}+\mu^{s}\right) \frac{\partial^{2} u_{s}}{\partial s^{2}}\right)-2 \frac{u_{s}}{R}+2 \frac{\partial u_{r}}{\partial s},
$$

where four sources of vorticity can be identified by each of the four terms on the right-hand side. As Gharib \& Weigand (1996) show in part, these are: (i) shear stress from the air (assumed to be negligible); (ii) surface tension gradients and acceleration/deceleration of the film itself; (iii) curvature of the surface (Lugt 1987); (iv) vertical motion of the surface which is characteristic of unsteady flows. Using equations from Gharib \& Weigand the vorticity flux of surface-parallel vorticity at the free surface is defined as

$$
v\left[\frac{\partial \omega_{z}}{\partial r}\right]_{r=0}=-\left(\frac{\partial u_{s}}{\partial t}+u_{s} \frac{\partial u_{s}}{\partial s}+\frac{1}{\rho} \frac{\partial p}{\partial s}+g \cos \theta\right)
$$

where the pressure is defined using Edwards et al. (1991) as

$$
p=p_{\text {ATM }}-\frac{\sigma}{R}+\frac{2 \mu^{s}}{R} \frac{\partial u_{s}}{\partial s}-\frac{\kappa^{s}+\mu^{s}}{R} \frac{\partial u_{s}}{\partial s} .
$$

The above equations show that surface-parallel vorticity can be generated and fluxed into the free surface by various mechanisms. These mechanisms will be discussed in terms of the components of vorticity observed for the surface-piercing cylinder wake.

\section{Experimental setup and procedure}

A small, recirculating water tunnel containing about 30 gallons of distilled water was used to generate the free-stream flow. The Plexiglas test section was 6 in. wide by $6 \mathrm{in}$. high by $30 \mathrm{in}$. long and the cylinder used was a smooth brass rod $3.175 \mathrm{~mm}$ in diameter.

In the contaminated surface cases, the surfactant chosen was sodium dodecyl sulphate (SDS) due to its easy accessibility and large range in surface tension versus concentration. In a review article, Chang \& Franses (1995) show that SDS has a range from $72 \mathrm{mN} \mathrm{m}^{-1}$ (that of pure water) at very low concentrations down to approximately $40 \mathrm{mN} \mathrm{m}^{-1}$ for higher surface concentrations.

The amount of surfactant was controlled by using a surface dam, upstream of which the surfactant collected. To control the placement of the Reynolds ridge (demarcation 

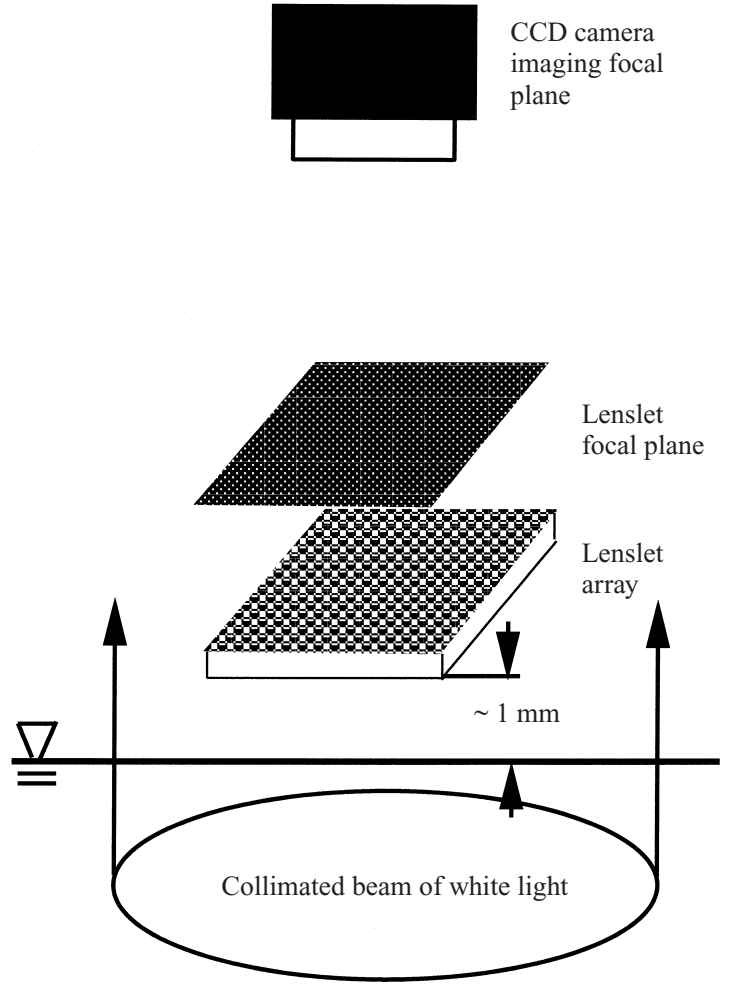

FIGURE 3. Schematic of the lenslet technique.

between the surfactant layer and clean water) additional surfactant was either added in solid form which dissolved on the free surface or removed through suction of the free surface. The resulting change in surface tension across the ridge was on the order of $5-10 \mathrm{mN} \mathrm{m}^{-1}$ (depending on the speed of the oncoming flow).

The other case studied was with a clean free-stream flow, but the cylinder itself was contaminated and shedding surfactant into the wake. To achieve this the cylinder was left piercing the free surface (which was completely covered with surfactant, as after the tunnel was turned off any surfactant on the free surface spread out uniformly) overnight and the surfactant would collect on the surface of the cylinder. Thus, a coating of surfactant molecules was present on the cylinder in the region near the free surface.

To examine the free surface in the wake of the cylinder two techniques were used. First, a simple shadowgraph system was set up where a 3 in. diameter beam of collimated light was generated and directed up through the bottom of the test section. A glass screen was placed over the test section onto which the light impinged after it passed through the free surface. A CCD camera was focused onto the screen to acquire greyscale images portraying the free-surface deformation.

The second technique, which utilized the same beam of collimated light, was a new lenslet technique as described by Roesgen, Lang \& Gharib (1998). This technique (schematic shown in figure 3) uses a lenticular or lenslet array placed just above the free surface and relies on the same principle as the shadowgraph technique - the bending of the light rays as they pass through a deformed free surface. Each lens in the array is $0.2 \mathrm{~mm}$ in diameter and has a focal length of $12 \mathrm{~mm}$. The CCD camera is 


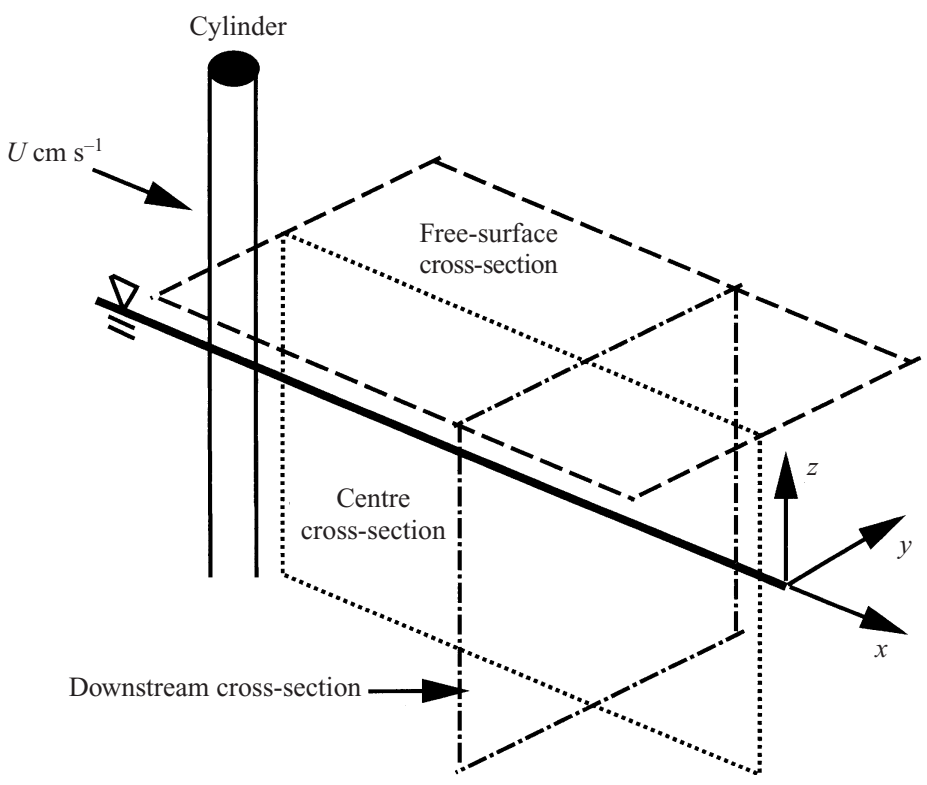

FIgURE 4. Schematic of the cylinder flow field and cross-sections studied.

focused onto the focal plane of the lenslet array in which the focal points (appearing as white dots on a black background in the digital image) move according to the free-surface slope. Measurement of the displacement of the array of focal points by an image-processing scheme results in a measurement of the free-surface deformation or height (where a least-squares two-dimensional integration is performed to transfer the slope data to height data). The sensitivity of the technique is found to be on the order of $10^{-4}$ radians. A more detailed description can be found in Roesgen et al. (1998). In both cases, an area of approximately $3 \mathrm{~cm}$ by $2 \mathrm{~cm}$ was studied.

Finally, digital particle image velocimetry (DPIV) was used to measure the velocity field in three orthogonal cross-sections of the flow as shown in figure 4, which defines a different coordinate system to that in figure 2. The DPIV technique is the same as presented by Willert \& Gharib (1991) and Weigand \& Gharib (1997). DPIV measures the velocity field by measuring the displacement of a random group of particles from one digital image to the next, where the time between the images is known. For this case the DPIV program was run with a $32 \times 32$ pixel window size and a step size of either 8 or 16 pixels. This resulted in a resolution of approximately $1 \mathrm{~mm}$ for the views studied. Since the accuracy of the technique is on the order of 0.1 pixels, this corresponds to an uncertainty of $1 \%$ for the velocity field and $3 \%$ for the corresponding calculated vorticity field.

The flow was seeded with $14 \mu \mathrm{m}$ silver-coated hollow glass spheres that are neutrally buoyant in water. The desired plane within the flow was illuminated using a Lexel Model 95 argon-ion laser with a power capability of 3 to $4 \mathrm{~W}$. A laser sheet was generated using a cylindrical lens, while mirrors were used to direct the sheet into the test section to study the desired cross-section of the flow. The three cross-sections studied were downstream, centre, and surface-parallel as labelled in figure 4. An area of the laser sheet was imaged through the use of a CCD camera to acquire the digitized images for DPIV. In the case of the surface-parallel cross-section, the area was imaged by acquiring the camera view from beneath the free surface using a $45^{\circ}$ 

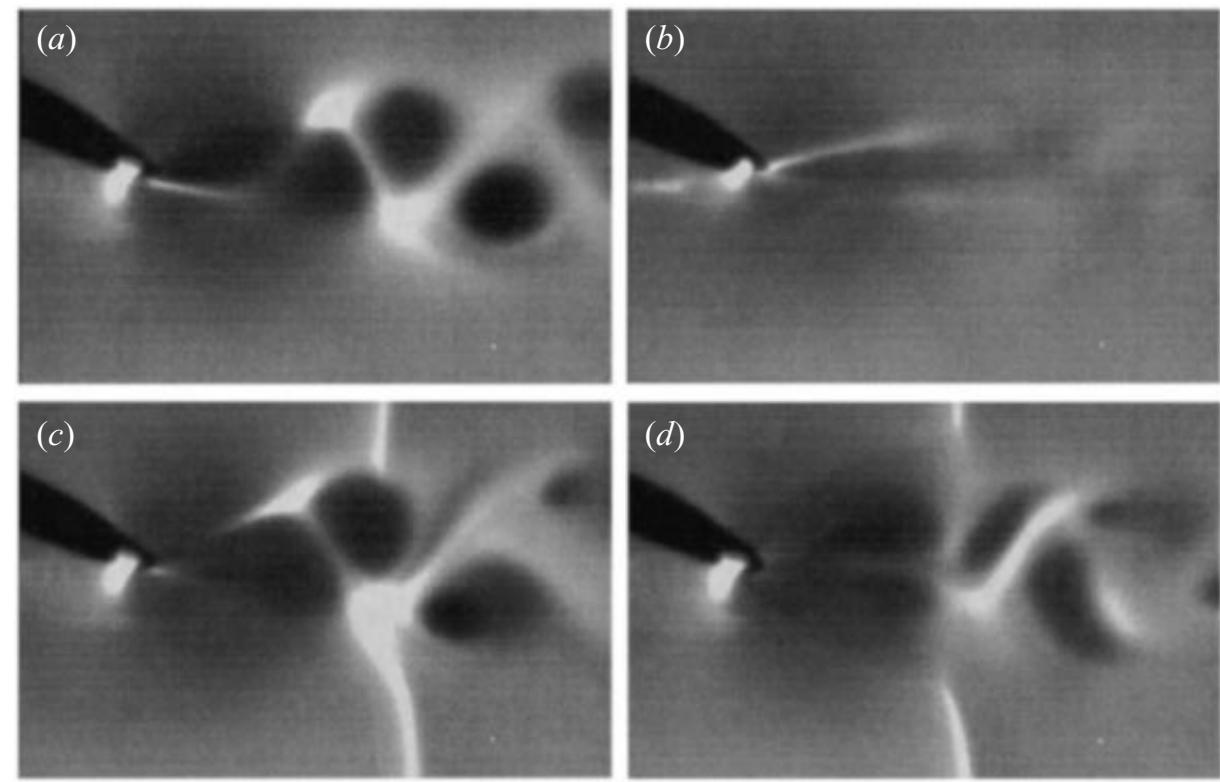

FIGURE 5. Shadowgraph images of a cylinder wake $(R e=460)$ where $(a)$ the surface is clean, $(b)$ the surface is contaminated, $(c)$ the ridge is behind cylinder (unattached), and $(d)$ the ridge is attached to the cylinder. (The ridge is the bright white line which appears vertically at the top and bottom of the image.)

mirror placed below the test section. Thus, no distortion of the data was allowed to occur as the imaged light did not pass through the deformed free surface. Also, in the case of the downstream cross-section, a downstream window of the tunnel was used to gain optical access to the desired illuminated plane. In addition for this case the sheet was thickened, using a cylindrical lens, from about $2 \mathrm{~mm}$ to $6 \mathrm{~mm}$ to allow the particles to remain illuminated during the exposure time of an image pair. The laser beam was shuttered using a Conoptics Model 303 optical shutter that was controlled by a General Pixels VTG-100 timing box. The camera provided the input signal to the timing box which generated a timing signal according to the programmed exposure and delay times for the shutter. The camera was run in continuous mode and the technique resulted in a sampling of the velocity field every $\frac{1}{15}$ th of a second.

\section{Results and discussion}

\subsection{Shadowgraph data}

Figure 5 presents shadowgraph images for a Reynolds number of 460, where the flow is moving from left to right. The area imaged is approximately $3 \mathrm{~cm}$ by $2 \mathrm{~cm}$, and four separate cases are shown. First, the surface is clean and as expected the vortices shed from the cylinder are observed as dark circles in the image. The larger deformation associated with a clean surface is observed, as no damping due to surface contamination is taking place. The second case is where the surface is contaminated (Reynolds ridge upstream of the cylinder) and little or no surface deformation is observed. This indicates that little or no coherent surface-normal vorticity is present at the surface.

The larger deformation field observed for the clean case is due to coherent, cylin- 
drical, vortical structures connecting at the free surface, that according to inviscid flow create a depression at the free surface where they connect. For the case of the contaminated cylinder, the wake image showed little surface deformation even though the ridge was far downstream of the cylinder. This indicated that the boundary layer or strain field behind the Reynolds ridge, that keeps the shedding filaments from connecting normally at the surface in figure $5(b)$, need not be present. Rather, only surface tension gradients, creating relatively large shear stresses due to the spreading of the surfactant, need exist to disrupt the consistency of the structures as they connect to the surface. The surface behind the cylinder may also be described as one with a spatially varying dynamic surface tension. The source of the surfactant from the cylinder is spreading out and trying to reach an equilibrium as it is convected downstream, and generating regions of high shear that, like the boundary layer of the Reynolds ridge, disrupt the connection process of the vortex filaments. Thus, being disrupted by this shear, they lack the coherency of the vortex filaments observed in the clean case and thus the ability to deform the surface to a larger degree.

The last two cases include a Reynolds ridge in the vicinity of the cylinder-in one case attached similarly to a bow shock in supersonic flow. Note that the shape of the ridge is highly dependent on the momentum of the flow beneath, with the tendency of the ridge to move forward where a lower-velocity flow is present. Thus in the unattached case the ridge takes the shape of the velocity profile in the wake. In these last cases one can observe what appears to be a stretching of the vortices as they pass through or behind the ridge. This stretching is evidence that the normal filaments are losing their coherency and tilting and disconnecting from the surface, thus redirecting the vorticity to the surface-parallel direction. Further proof of this occurrence will be discussed in the next sections.

\subsection{Surface-parallel cross-section velocity data}

Figure 6 shows a sequence of three velocity/vorticity measurements where the surface is clean and the cross-section studied is at the free surface. In all figures such as figure 6, where the velocity data are shown, the cylinder is located just to the left of the figure (or such that the right far edge of the cylinder is located at $x=0$ ). As observed in the shadowgraph, one sees the surface-normal vortices shed from the cylinder and convecting downstream. On the other hand, for the contaminated case in figure 7, where the ridge is upstream of the cylinder, the wake is greatly altered. Here it almost appears as a very low Reynolds number wake where low vorticity levels are characteristic of the weak surface deformation observed in the shadowgraph results.

Next, figure 8 shows a velocity/vorticity sequence where the ridge is in the near wake of the cylinder. One can see the drop in velocity as the flow meets the ridge, and the stretching of the vorticity field as the filaments pass through the ridge. The circulation of various vortex filaments was measured from the vorticity data using a DPIV program where a closed curve of constant vorticity level is defined around a structure and the circulation calculated. Several vortices were chosen from each run and circulation calculated as the flow progressed. These normalized data are presented in figure 9 and show a loss of circulation in some cases in excess of $80 \%$ as the filaments pass through the ridge, while that observed due to dissipation in the clean case is minimal (on average only $10 \%-20 \%$ loss).

Finally, figure 10 shows a sequence where the oncoming flow is clean but the cylinder is contaminated and shedding surfactant into the wake. In this case the presence of surfactants causes an area of lower surface velocity in the line of sight directly behind the cylinder, which meanders and is quite unsteady. The lower velocity 


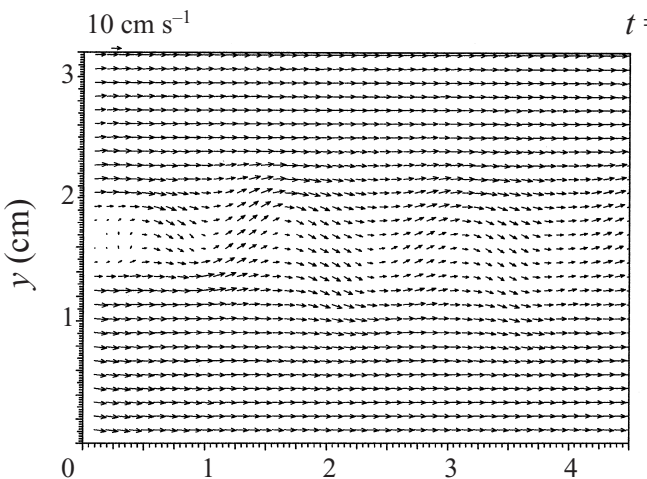
$t=0 \mathrm{~s}$
$10 \mathrm{~cm} \mathrm{~s}^{-1}$ $t=0.067 \mathrm{~s}$
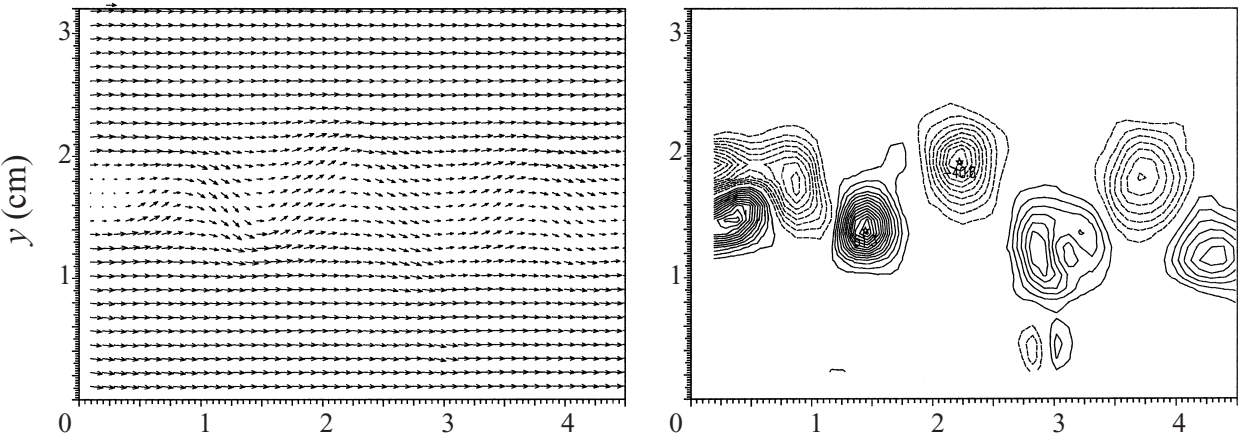

$10 \mathrm{~cm} \mathrm{~s}^{-1}$ $t=0.133 \mathrm{~s}$
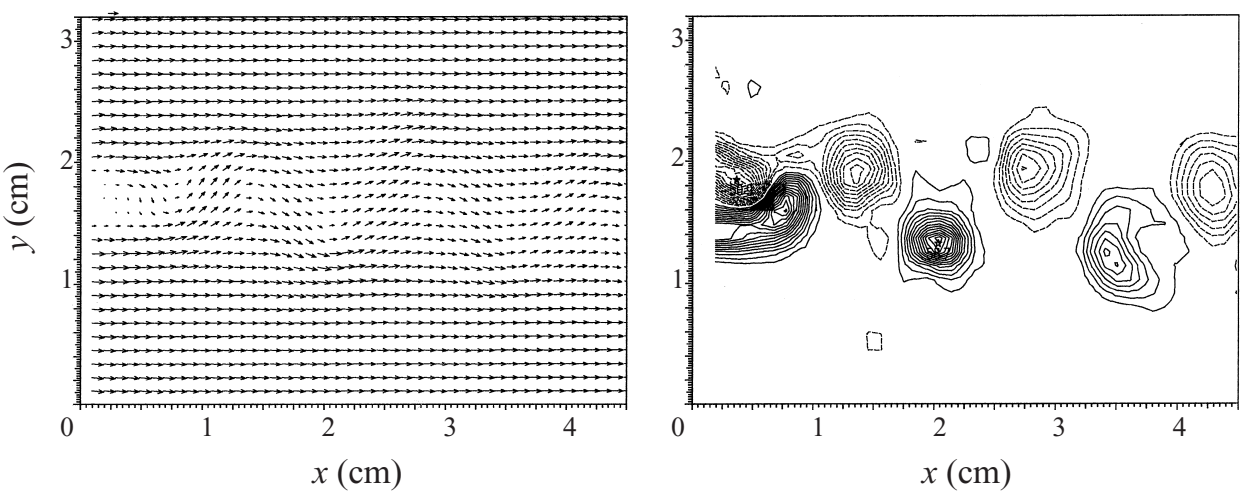

FIGURE 6. Sequence of velocity and vorticity plots in the $(x, y)$-plane for a clean free surface and $R e=350$. Vorticity contour levels $\left(\mathrm{s}^{-1}\right)-4,-8,-12, \ldots$ (dashed lines) and $4,8,12, \ldots$ (solid lines).

and high surface tension gradients, not to mention the spreading of the surfactant in the transverse direction as it enters the wake, all contribute in a complex manner to make the flow incoherent with wide more random distributions of vorticity. However, it is evident that all these mechanisms account for the decrease in surface deformation that has been observed for this case. In particular, without the presence of larger surface-normal coherent structures at the surface as in the clean case, the surface deformation is minimal as previously observed.

A short sequence of simultaneous velocity/vorticity and surface deformation measurements are shown in figure 11 . Here the new lenslet technique was used to acquire 

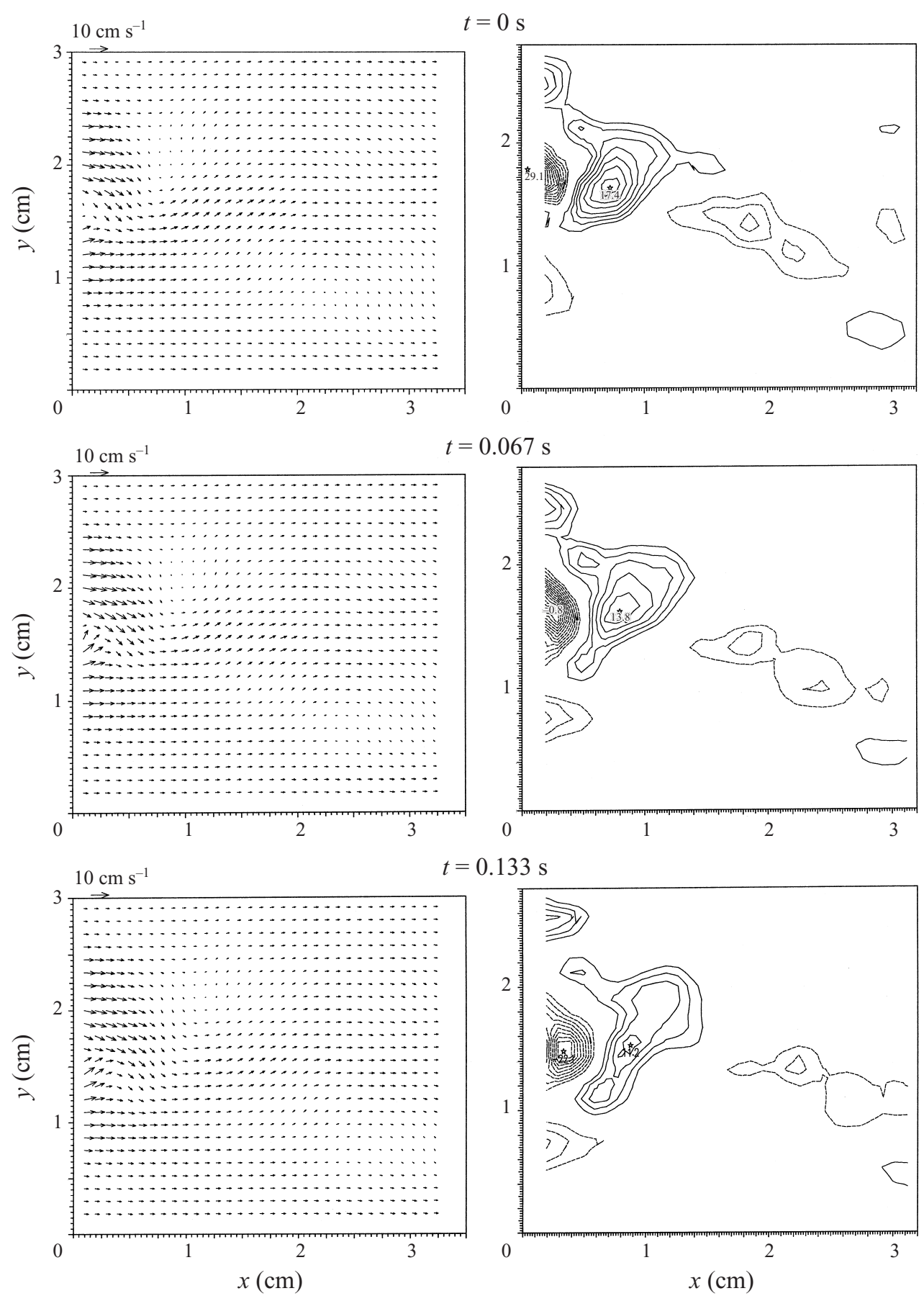

FigURE 7. Sequence of velocity and vorticity plots in the $(x, y)$-plane for a contaminated free surface and $R e=350$. Vorticity contour levels $\left(\mathrm{s}^{-1}\right)-4,-6,-8, \ldots$ (dashed lines) and $4,6,8, \ldots$ (solid lines).

the surface height profile. This sequence shows the attachment process of the ridge to the cylinder. First, the shape of the ridge when attached follows the outline of the vorticity behind the cylinder, or where the flow is decelerated in the circulating region. Again, the stretching of the vortices due to the almost stagnant surfactant film is 


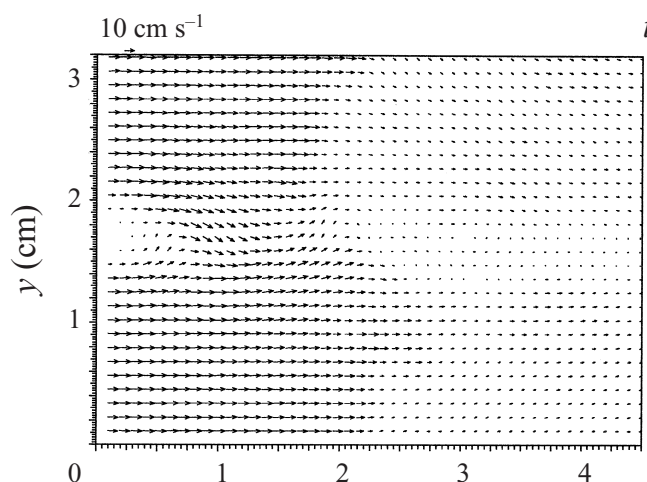
$t=0 \mathrm{~s}$
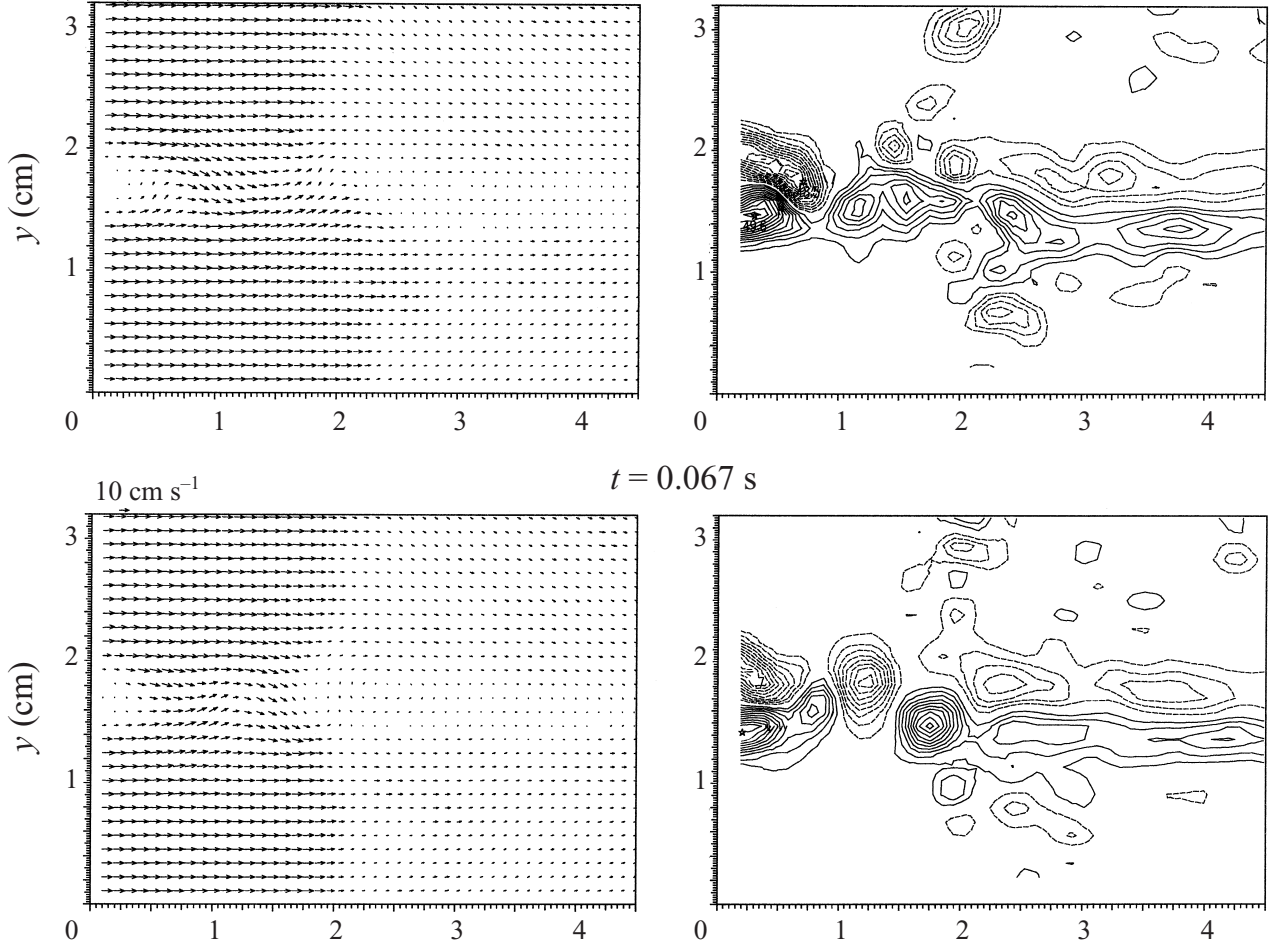

$t=0.067 \mathrm{~s}$

$10 \mathrm{~cm} \mathrm{~s}^{-1}$

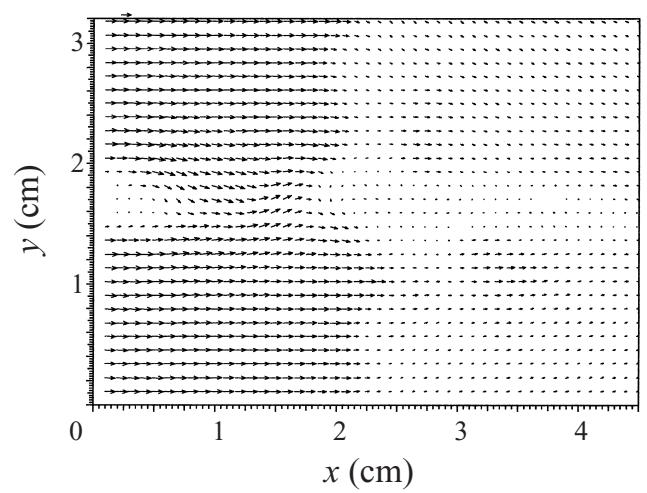

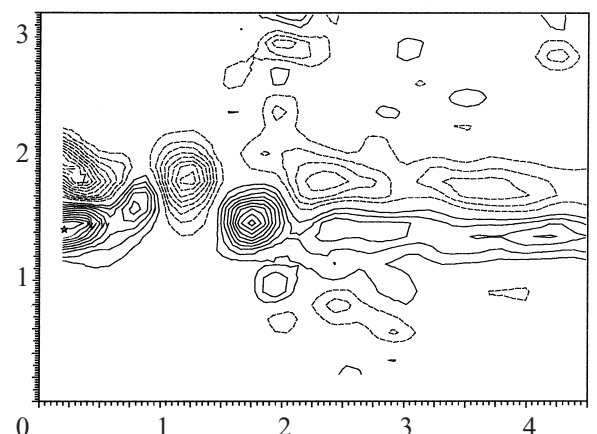

$t=0.133 \mathrm{~s}$

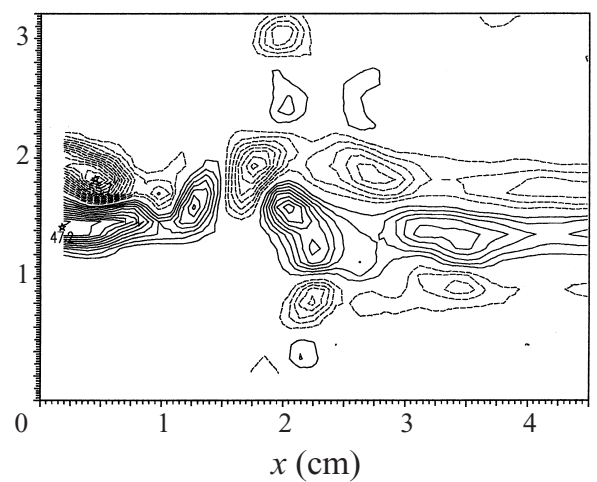

FIGURE 8 . Sequence of velocity and vorticity plots in the $(x, y)$-plane where the ridge is in the wake of the cylinder and $R e=350$. Vorticity contour levels $\left(\mathrm{s}^{-1}\right)-4,-8,-12, \ldots$ (dashed lines) and 4,8 , $12, \ldots$ (solid lines).

observed. Also, the surface deformation is highly dependent on the coherent normal vorticity present at the free surface. What in fact occurs during the attachment process is that initially the surface deformation behind the ridge is minimal, but in the small time as the ridge attaches to the cylinder the film is stretched over a small region. As this expansion of the film occurs the concentration of surfactant is decreased, and thus also the magnitude of the surface tension gradients over this small area. Thus in the later figures for this case, one sees greater evidence of surface-normal vorticity associated with the shedding filaments in the vorticity and surface deformation plots. 

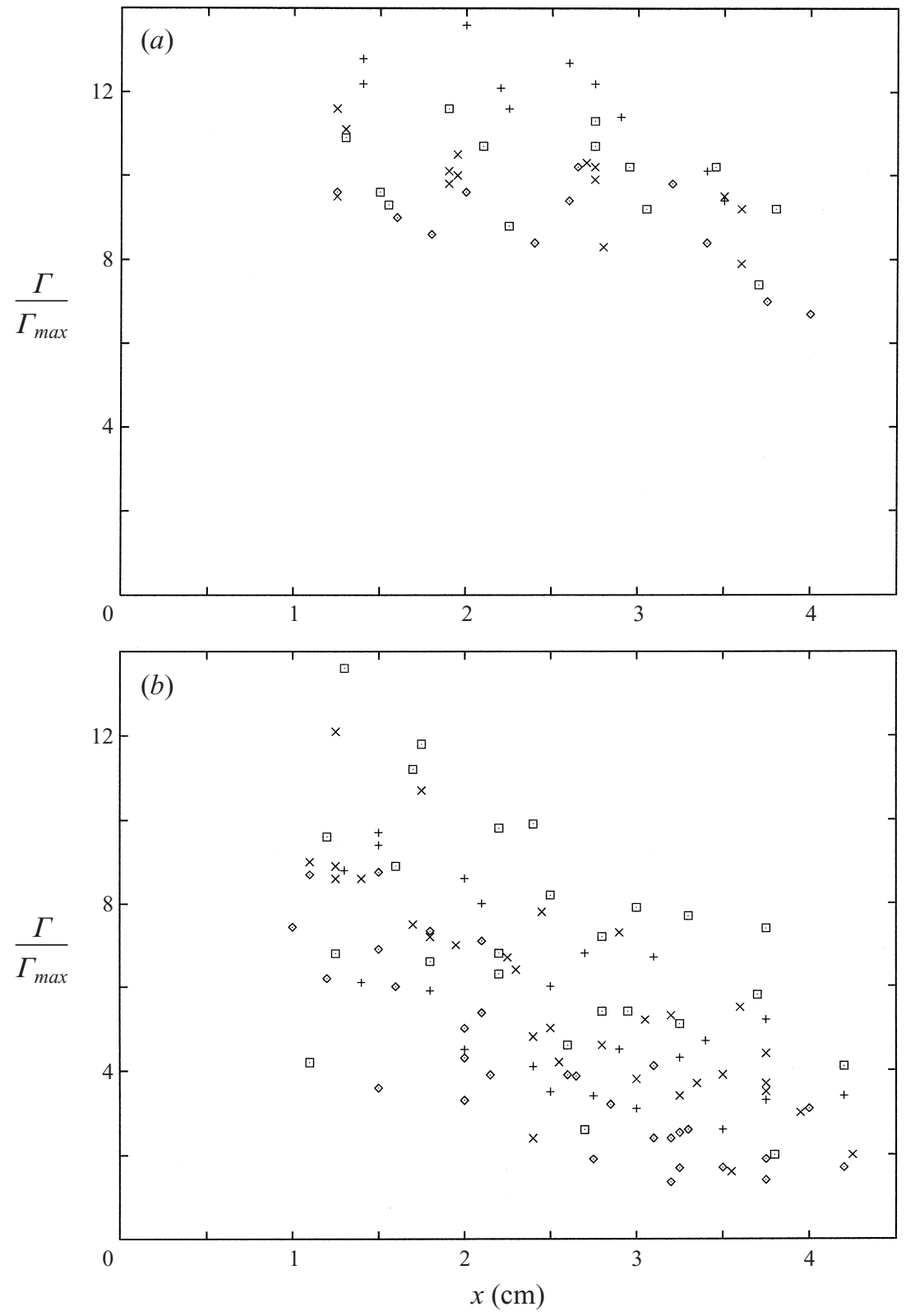

FIGURE 9. Plots of circulation in the free-surface cross-section ( $y$-axis multiplied by 10 and normalized with respect to the average maximum circulation observed for each run) for shedding vortex filaments at various downstream distances: $(a)$ a clean free surface, and $(b)$ for the case where the ridge is sitting at approximately $x=1.5 \mathrm{~cm}$.

However, the amount of surface-normal vorticity will decrease again as the film spreads forward into this expanded area.

Additional data were taken for the contaminated case at two cross-sections located at $0.55 \mathrm{~cm}$ (about 1.7 cylinder diameters) and $1.10 \mathrm{~cm}$ (about 3.5 cylinder diameters) below the free surface. Velocity and vorticity field sequences for these two cases are shown in figure 12. These results show the increasing similarity in the wake has to 

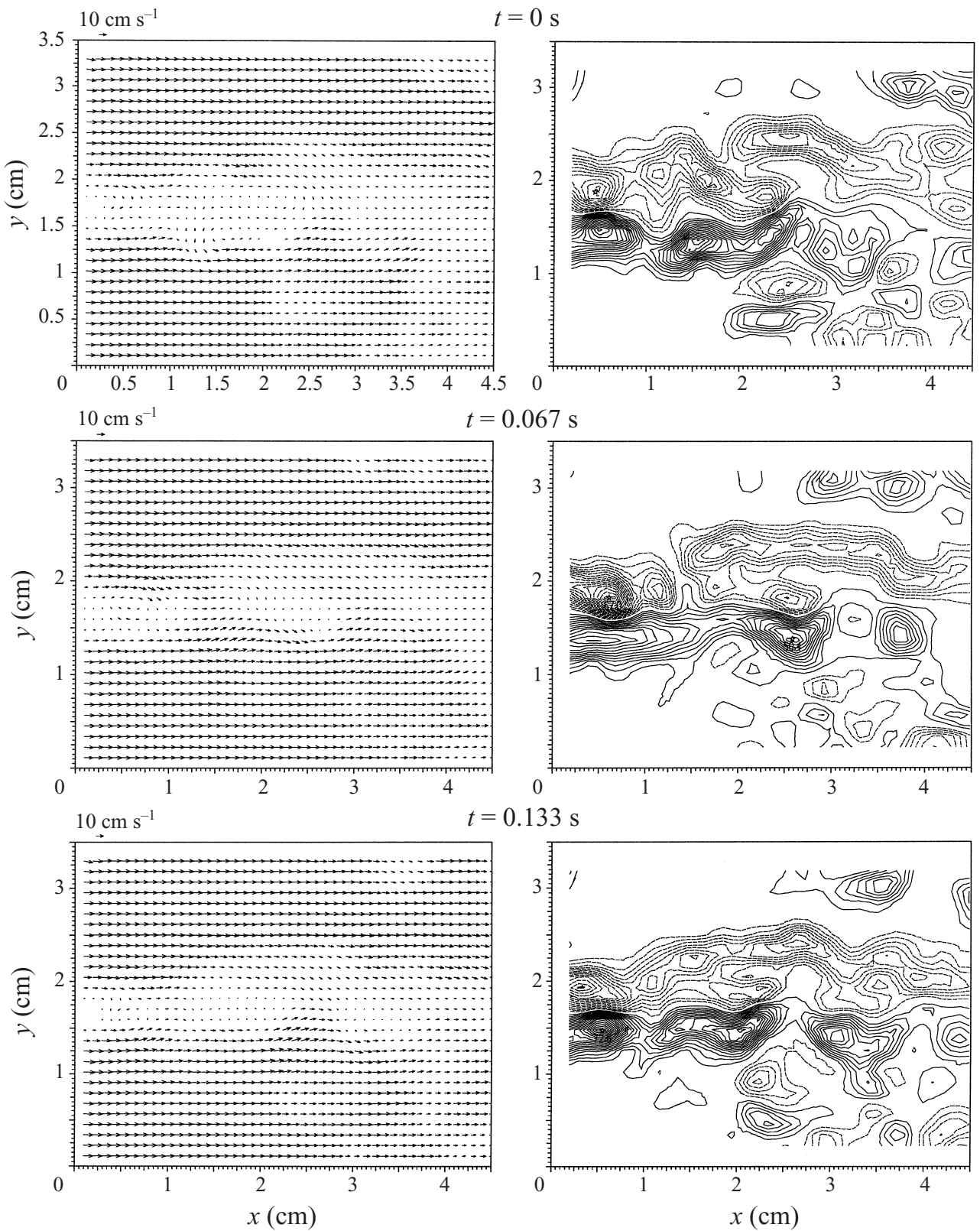

FIGURE 10. Sequence of velocity and vorticity plots in the $(x, y)$-plane where the cylinder is contaminated and $R e=410$. Vorticity contour levels $\left(\mathrm{s}^{-1}\right)-4,-8,-12, \ldots$ (dashed lines) and 4,8 , $12, \ldots$ (solid lines).

that of the clean case or the standard von Kármán vortex street farther below the free surface. This is of course due to the fact that any free-surface effects are decreased the farther one moves away from the boundary. Note that both these cases are below the thickness of the boundary layer. The middle cut portrays a stretching of the vortex filaments while still attached to the cylinder, similar to that observed behind the ridge in the case of figure 8 . The bottom cut appears to show a pairing occurring 

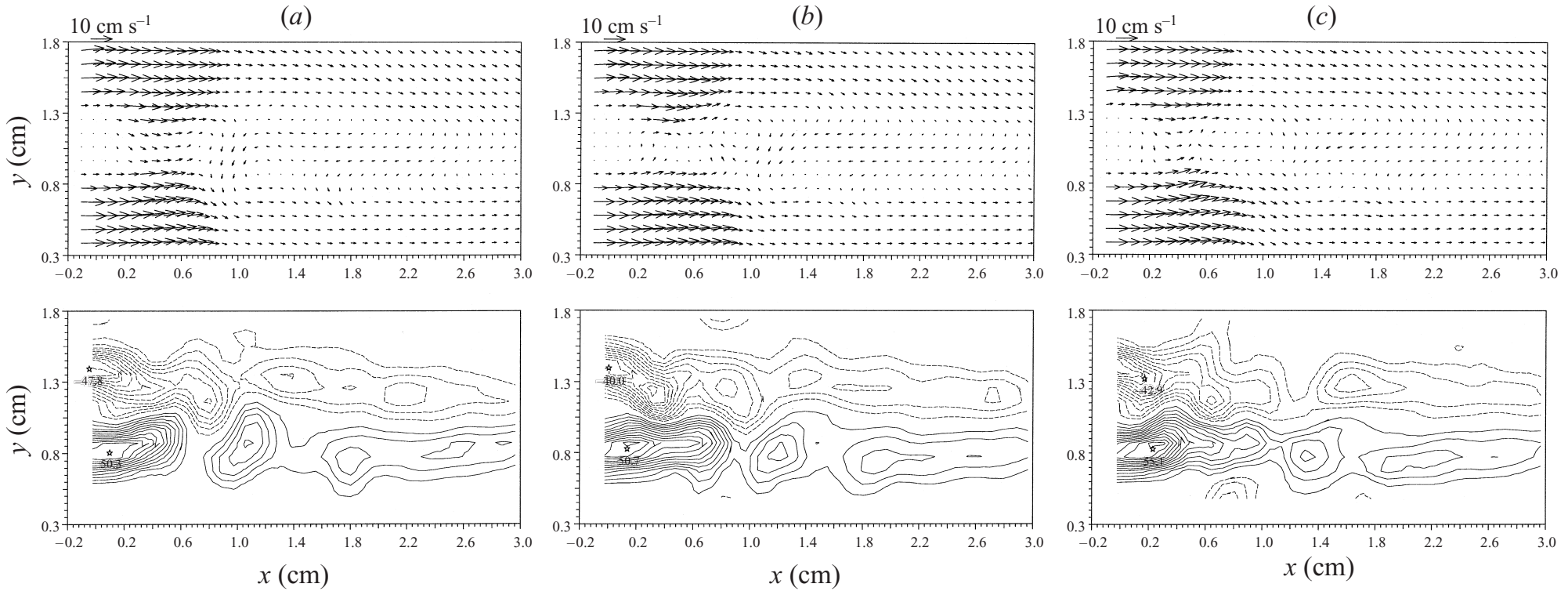

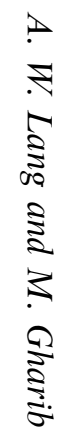
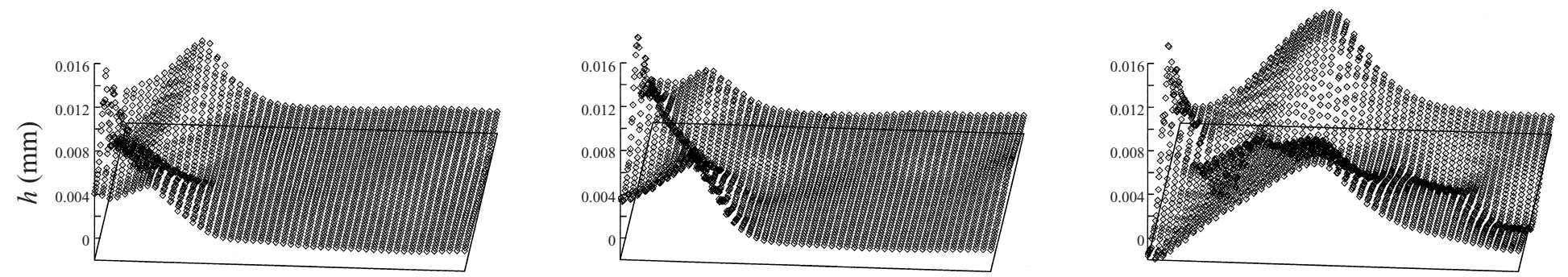

FIGURE $11(a-c)$. For caption see facing page. 

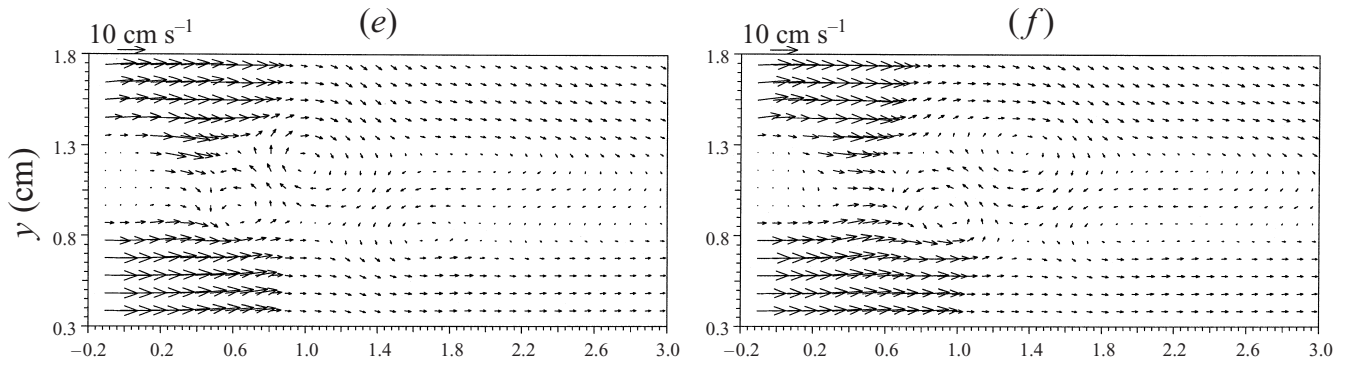

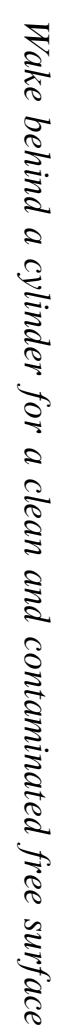

Figure 11. Time sequence of simultaneous $(R e=350)$ velocity and vorticity (contour levels $\left(\mathrm{s}^{-1}\right)-4,-8,-12, \ldots$ (dashed lines) and $4,8,12, \ldots$ (solid lines)) and free-surface height measurements showing the attachment process of the ridge to the cylinder. 


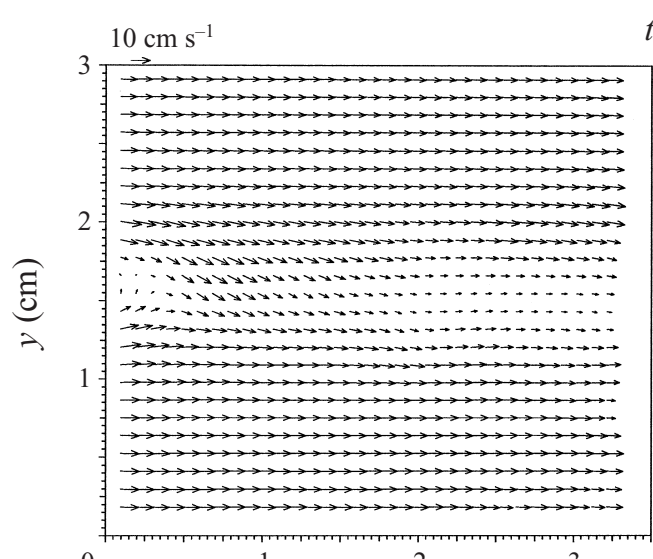

(a)
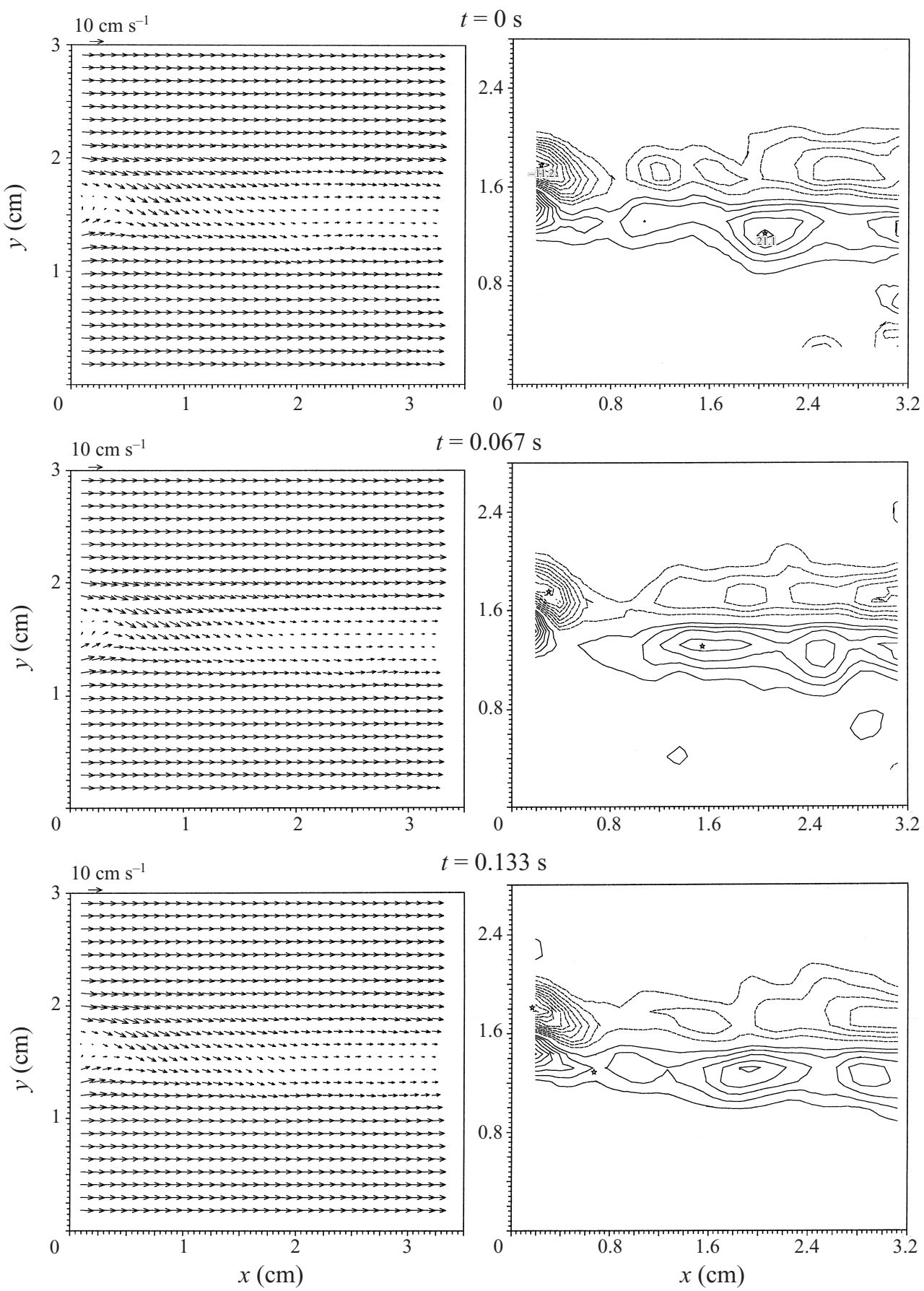

$=0.133 \mathrm{~s}$

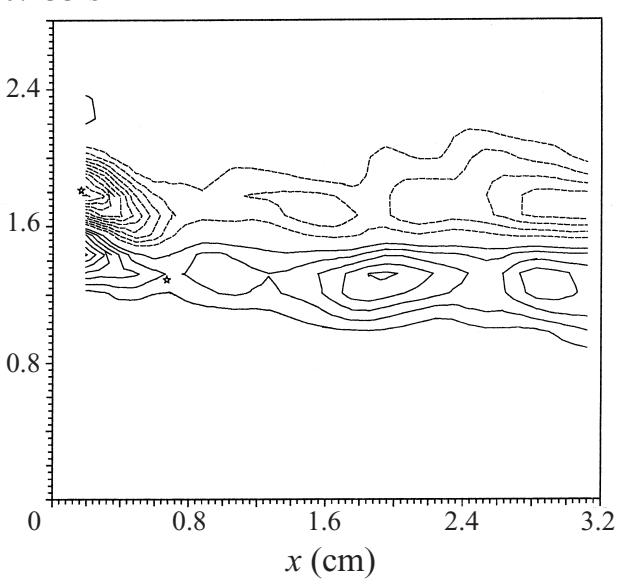

FIGURE $12(a)$. For caption see facing page 
(b)
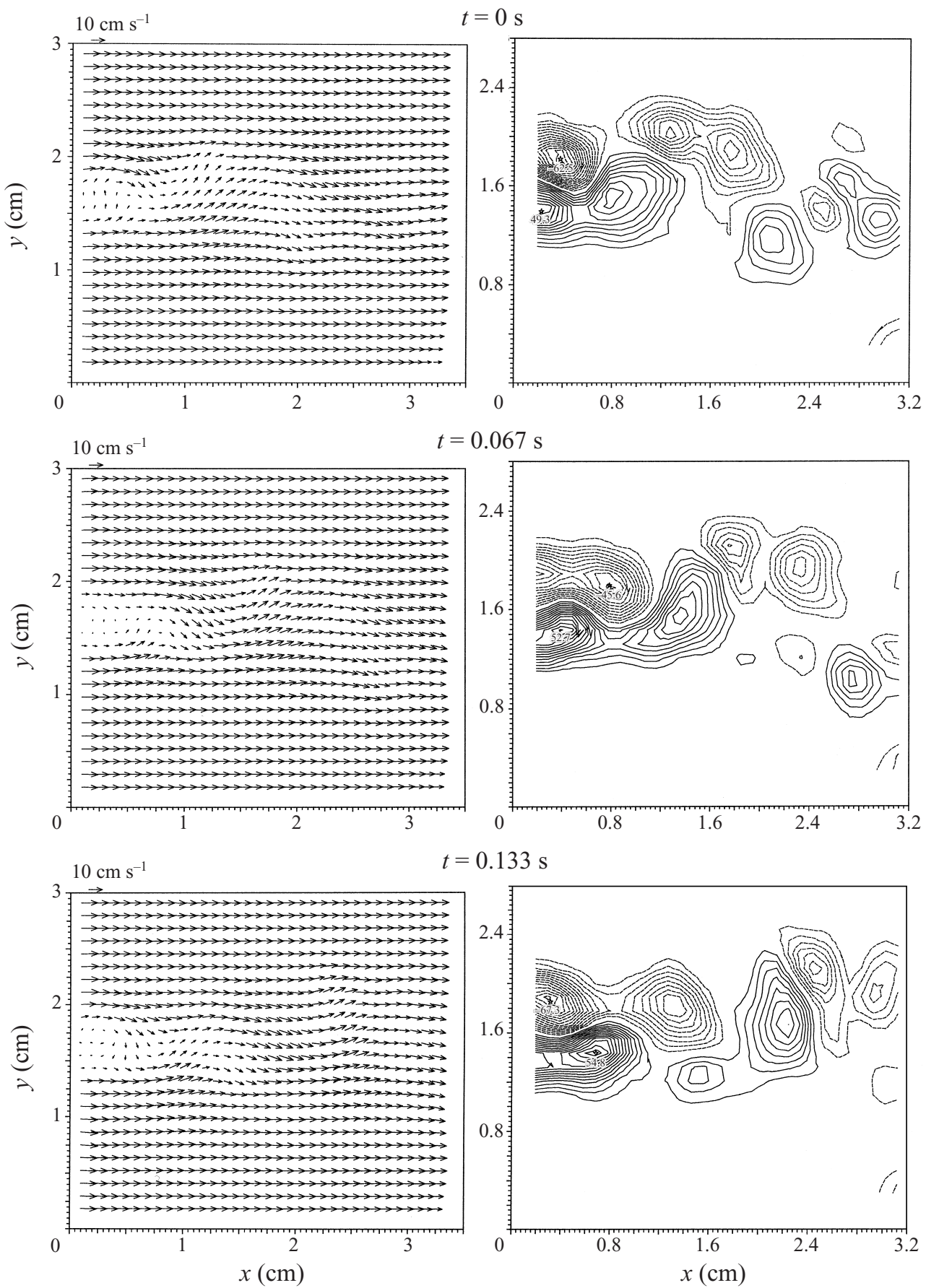

$=0.133 \mathrm{~s}$

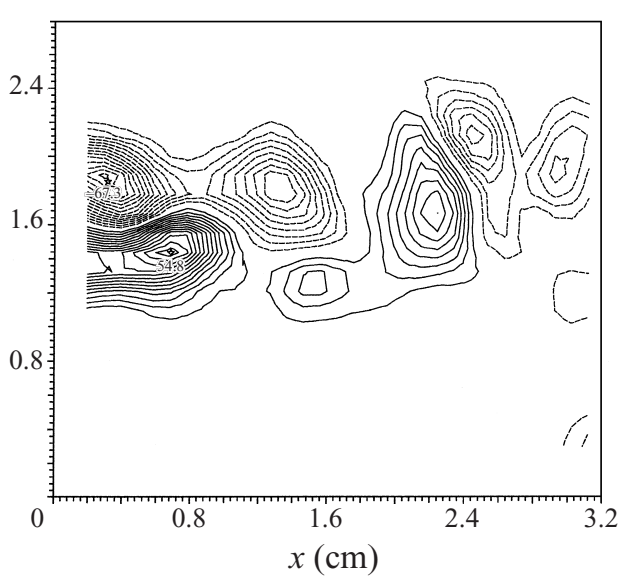

FIGURE 12 . Sequence of velocity and vorticity plots in the $(x, y)$-plane where the surface is contaminated and $R e=350$ at $(a)$ a cut $0.55 \mathrm{~cm}$ and $(b)$ a cut $1.10 \mathrm{~cm}$ below the free surface. Vorticity contour levels $\left(\mathrm{s}^{-1}\right)-4,-8,-12, \ldots$ (dashed lines) and $4,8,12, \ldots$ (solid lines). 

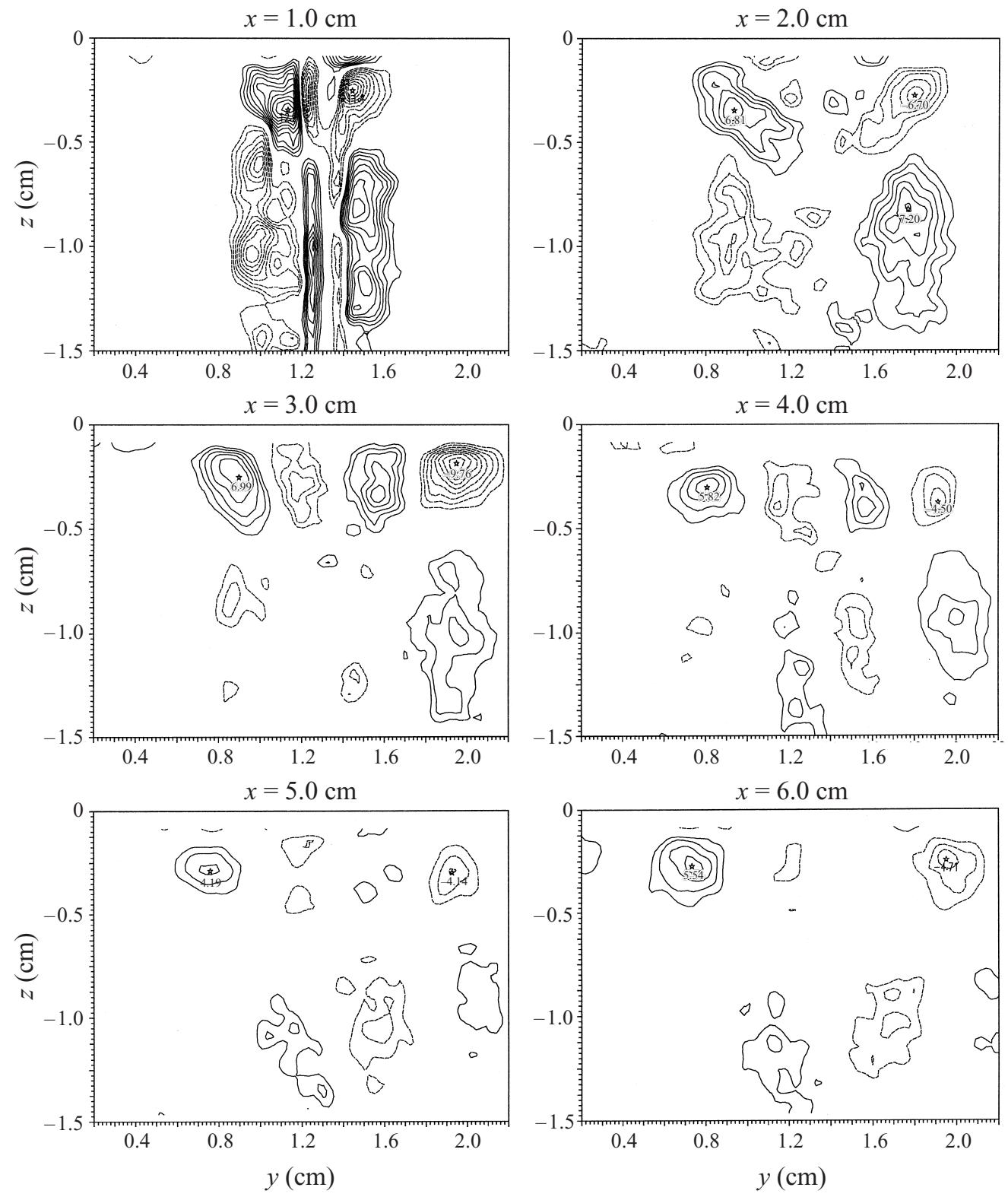

FIGURE 13. Six different downstream vorticity plots ( $y, z$-plane) where $x$ is distance downstream of the cylinder each averaged over 500 velocity files. $R e=410$ and the surface is clean. The free surface is at $z=0$. Vorticity contour levels $\left(\mathrm{s}^{-1}\right)-2,-3,-4, \ldots$ (dashed lines) and $2,3,4, \ldots$ (solid lines).

between the opposite-sign filaments and this will be explained later in the model to be presented.

\subsection{Downstream cross-section velocity data}

In this cross-section, the plane illuminated is one where the free-stream flow is perpendicular to the cross-section and coming out of the page. To examine the flow for this case an averaging was performed where the results shown used 500 sequential 


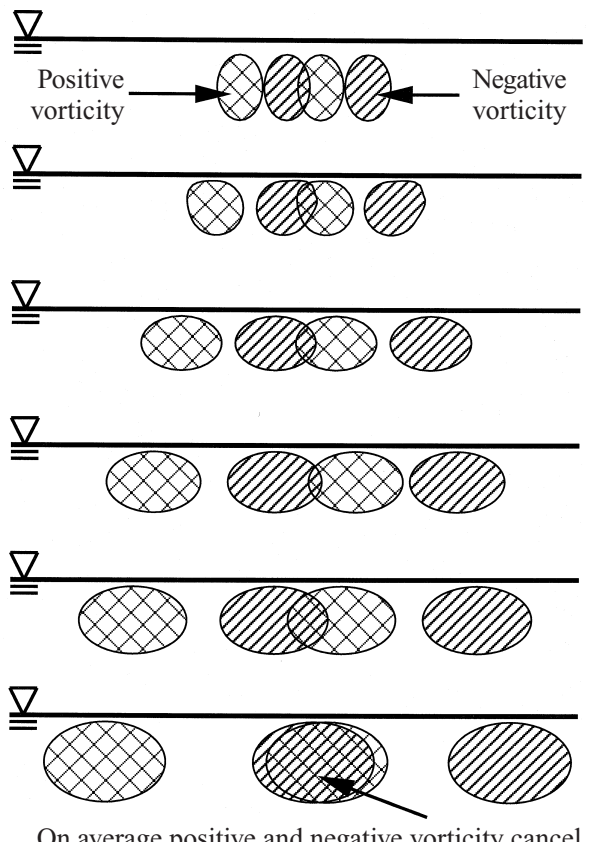

FIGURE 14. Schematic of the six downstream distances of figure 13 (top to bottom $x$ increasing) for the clean case showing the placement of the zigzag vortex pair.

velocity files to calculate the data. Due to the orientation of the shedding vortex filaments, with the primary vorticity component not in the plane being studied but instead passing through the cross-section, instantaneous velocity data gave little useful information. Thus, the resulting vorticity plots presented were calculated from the averaged velocity data.

Figure 13 shows the vorticity plots obtained from the averaged data at various distances downstream of the cylinder for the case where the surface is clean. A surface-parallel component of vorticity is observed near the free surface, which at first glance appears to be two vortex pairs. However, upon further inspection it was realized that it is in fact one vortex pair that is moving from side to side within the wake. This pair is located on the inside edge of each shedding vortex filament and thus creates a zigzag pattern when viewed from above. This pattern is also visible in the shadowgraph visualization of figure $5(a)$ as the bright region in the centre of the wake that zigzags between the dark vortex filaments. In the shadowgraph, bright regions correspond to an upwelling of the flow at the surface, and this agrees with the sign of the zigzag vortex pair observed. The placement of the pair is dependent on both the growth of the wake and the growth of the separation between the vortex pair as it interacts with the free surface. A visual explanation of this is shown in figure 14 , where in some cases, such as the one farthest downstream, a cancelling of the vorticity is observed due to the averaging performed. Thus, because of the unsteady nature of the flow and the movement of the pair within the cross-section studied, at times the zigzag vortex can appear as one pair with twice the width of separation.

Next, data are presented in figure 15 for two different Reynolds numbers where the ridge is unattached to the cylinder in the wake yet upstream of the cross-section studied (i.e. ridge at $2 \mathrm{~cm}$ downstream and cross-section $4 \mathrm{~cm}$ downstream of cylinder). 

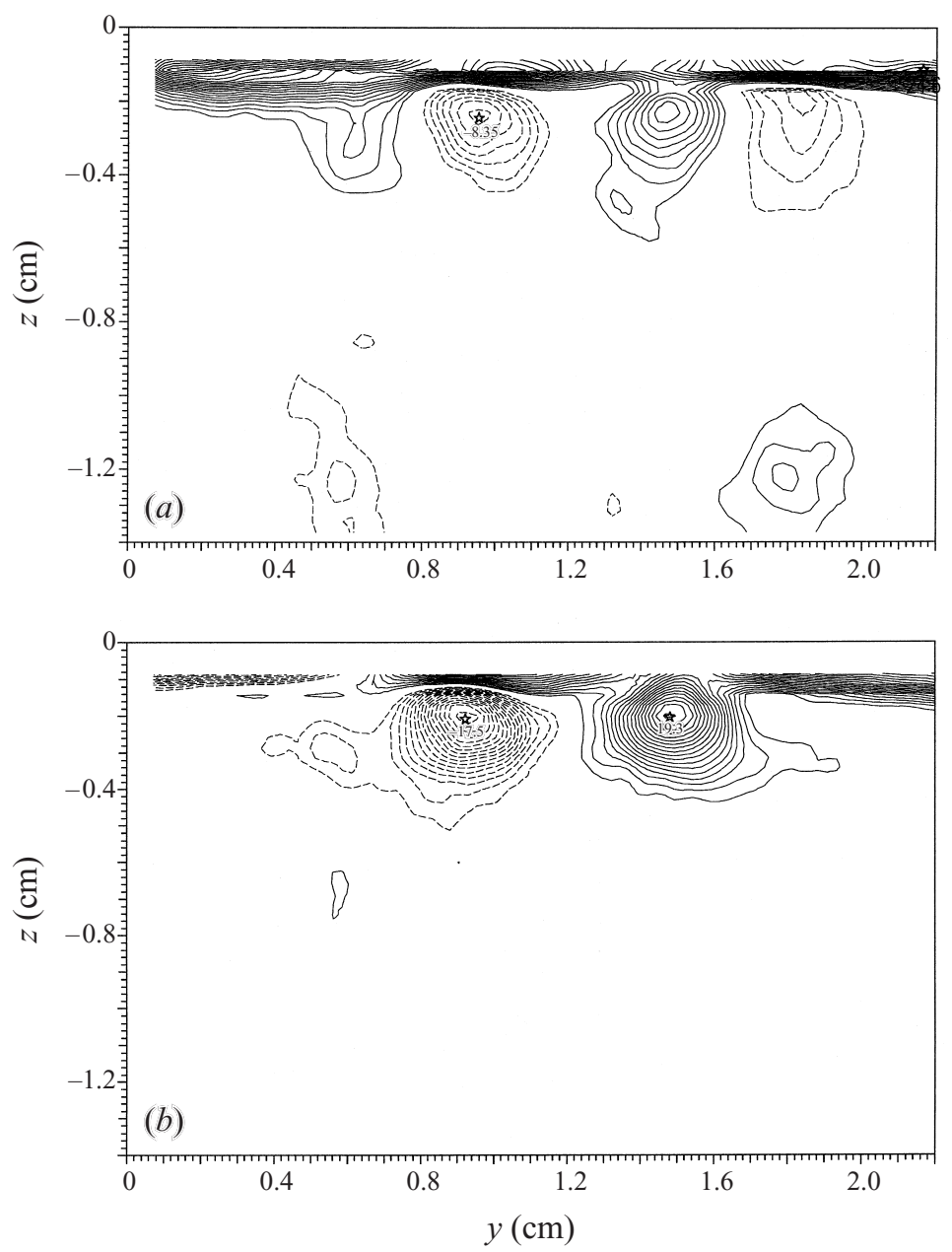

FiguRE 15 . Vorticity plots (contour levels $\left(\mathrm{s}^{-1}\right)-2,-3,-4, \ldots$ (dashed lines) and $2,3,4, \ldots$ (solid lines)) in the $(y, z)$-plane located at $x=4 \mathrm{~cm}$ downstream of the cylinder where the ridge is located at $x=2 \mathrm{~cm}$ (unattached) and thus upstream of the cross-section for $(a) R e=350$ and $(b) R e=410$. The free surface is at $z=0$.

The two cases show that, for the lower Re, the same four vortex structures are present as in the clean case; however the inner pair is greater in strength. For the higher $R e$, only the inner pair is present. The inner pair in this case corresponds not to the zigzag vortex observed for the clean case but rather to a conversion of the vorticity in the shedding vortex filaments themselves to the surface-parallel component. The higher Re case corresponds to a stronger Reynolds ridge and thus larger surface tension gradient or shear stress at the free surface. This also leads to reduced surface deformation. However, for a lower $R e$ the weaker ridge does not have as strong a tendency to turn the filaments to the surface-parallel direction, and thus signatures of both components are observed.

Before continuing further, the vorticity layer at the free surface (seen in figure 15) should be explained. This vorticity is produced due to the secondary flow that is generated in the monolayer film itself. The formation of the boundary layers on the sidewalls of the tunnel reduces the momentum of the flow beneath the sides of the 
(a)
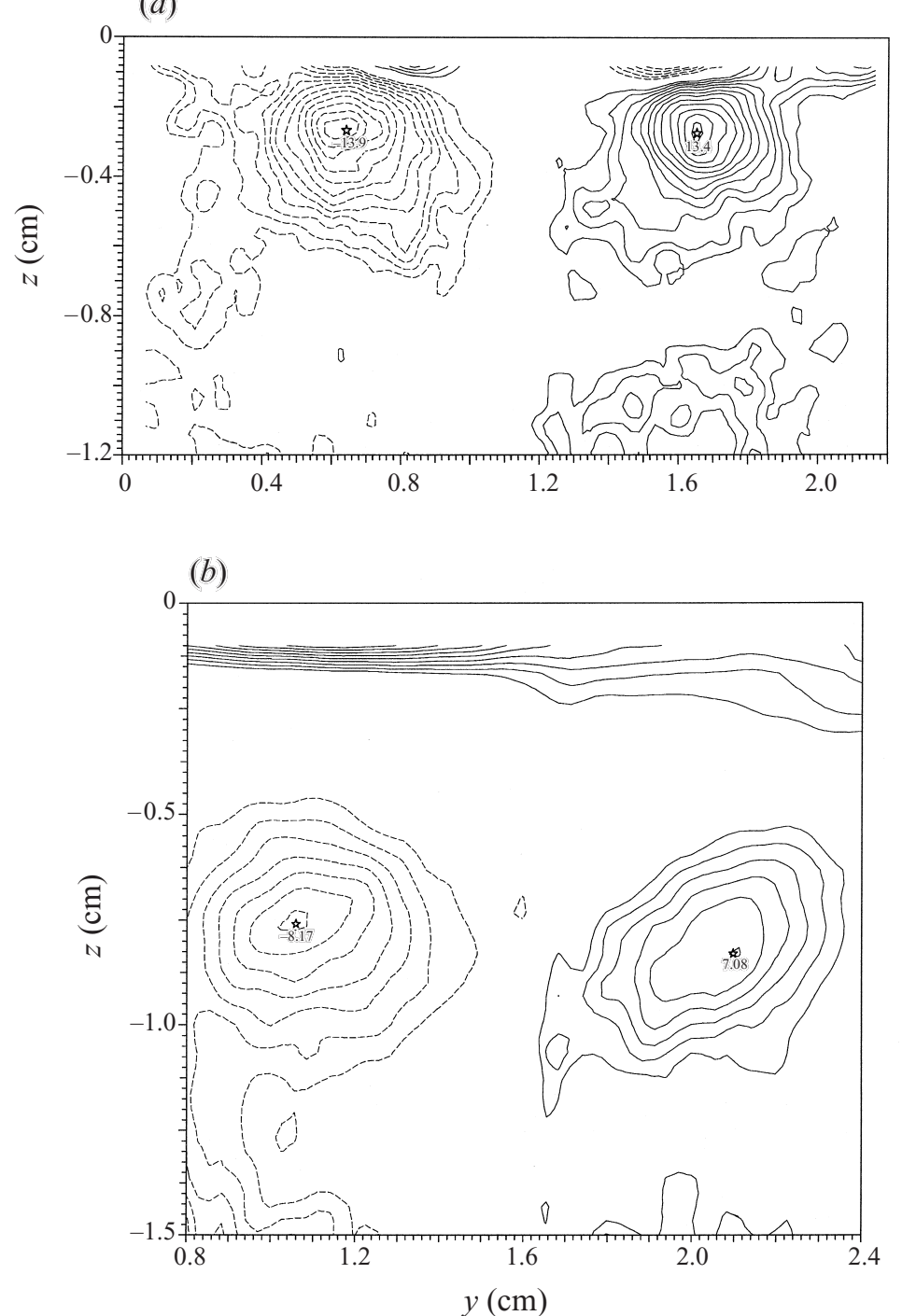

FIGURE 16. Vorticity plots (contour levels $\left(\mathrm{s}^{-1}\right)-2,-3,-4, \ldots$ (dashed lines) and $2,3,4, \ldots$ (solid lines $))$ in the $(y, z)$-plane $(R e=410)$ located at $(a) x=4.0 \mathrm{~cm}$ where the cylinder is contaminated and at $(b) x=3.75 \mathrm{~cm}$ where the surface is contaminated (ridge upstream of the cylinder). The free surface is at $z=0$.

surfactant film at the walls of the tunnel. This results in a slight upstream velocity at the walls and a downstream velocity of the film at the centre. This recirculating flow has been observed by both Scott (1982) and Kenning \& Cooper (1966). Depending on the placement of the ridge with respect to the centreline of the tunnel, different components of this secondary flow are observed in the vorticity layer at the surface. The experiments were performed at different locations with respect to the centreline and no effect was observed on the flow field itself; rather the secondary flow acts more like a 'background' vorticity that is pasted onto the flow field.

The last two cases to be considered show similar results. The case where the oncoming surface flow is clean while the cylinder is contaminated as well as the case 
(a)

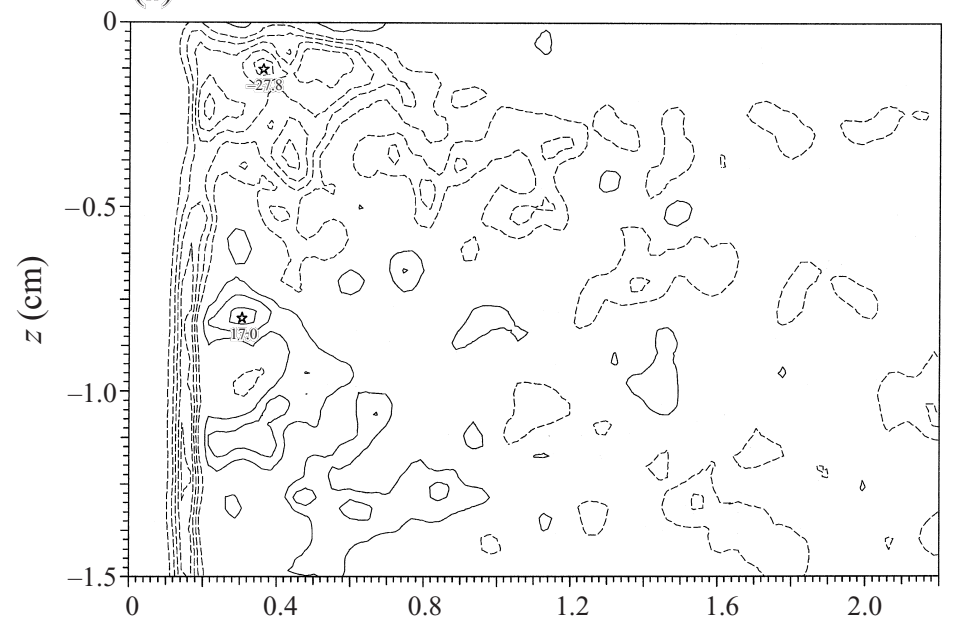

(b)

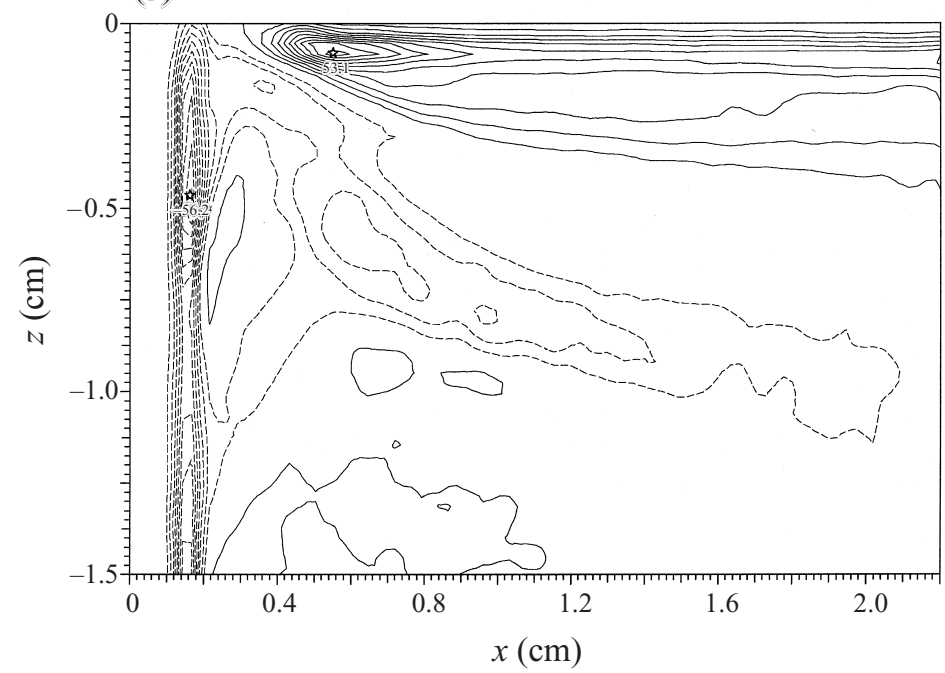

FigURE 17. Vorticity plots (contour levels $\left(\mathrm{s}^{-1}\right)-5,-10,-15, \ldots$ (dashed lines) and $5,10,15, \ldots$ (solid lines)) where $R e=350$ in the $(x, z)$-plane and $(a)$ the surface is clean and $(b)$ the surface is contaminated. The edge of the cylinder is on the left edge of the plot at $x=0$ and the free surface is at $z=0$.

where the surface is contaminated (i.e. ridge about $7 \mathrm{~cm}$ upstream of the cylinder) are shown in figure 16. The structures observed in figure $15(b)$ are again seen, however stronger in the contaminated cylinder case. The other difference is that in the contaminated case the boundary layer that forms at the Reynolds ridge interacts with the flow field and pushes the vortex pair deeper beneath the free surface.

\subsection{Centre cross-section velocity data}

For this cross-section, again an averaging of 500 velocity files was performed. The vorticity data for the clean surface and contaminated surfaces are shown in figure 17. Note for the contaminated case the higher levels of vorticity as well as the boundary layer at the surface due to the Reynolds ridge upstream of the cylinder. 
It is interesting to observe the shear layer of negative sign that is formed beneath the boundary layer for this case. Note that the depth of maximum vorticity for this shear layer corresponds to the middle cut shown in figure $12(a)$ or $0.55 \mathrm{~cm}$ below the surface. The clean case shows some vorticity just behind the cylinder (where the back edge of the cylinder is located at $x=0$ ) but no coherent surface-parallel structures downstream in this cross-section. That data were not taken for the other cases at this cross-section due to the unsteady placement of the ridge within the test area during the acquisition of the 500 images. However, these two cases give enough insight to suggest the flow models to be presented in the next section.

\section{Discussion of flow models}

First, for the clean case a model of the flow is presented in figure 18(a). This schematic shows the von Kármán vortex street characteristic of this low Reynolds number flow field. However, the interaction of the shedding vortex filaments, which must connect normally, with the free surface creates regions of high surface curvature as evident in the shadowgraph visualizations. The bright zigzag region between the shedding filaments corresponds to a region convex in surface deformation or an upwelling of flow. This agrees with the sign of the zigzag vortex pair observed in the velocity data, and the placement of this pair is shown in the model. Thus, the cylinder wake in the case of a clean surface shows the characteristics of large surface deformations due to the normally connecting shedding vortex filaments. This high curvature at the surface generates a vortex pair of the surface-parallel component that curves between the shedding vortex filaments and convects downstream with the flow. Thus, there is evidence that surface-parallel vorticity is generated due to surface deformations or surface curvature. However, here the vorticity is a result of the surface-normal vortex filaments deforming the surface in the wake, rather than that generated by surface tension gradients which interferes with and alters the connection of the filaments to the free surface.

For the cases of the contaminated surface, and the contaminated cylinder met by an oncoming clean free surface, quite a different scenario was observed and this model is shown in figure 18(b). Similar to the case where the boundary is solid, a kind of horseshoe vortex system was observed, the only difference in this case being that surface-normal vorticity is allowed to persist at the boundary due to the contaminated surface condition. Thus the horseshoe connects to the corresponding regions of vorticity at the surface behind the cylinder, rather than looping around and connecting in front of the cylinder. In the case where the ridge is upstream of the cylinder the boundary layer creates a region of high shear at the surface. This has the effect of pushing the horseshoe vortex deeper into the flow. However, in the case of the contaminated cylinder the interference of the boundary layer is not present and some significant components of surface-normal vorticity are observed. However, on average the conversion of the vorticity, in the vicinity of the free surface, to the surface-parallel component is observed by the creation of the horseshoe vortex exactly below the free surface. In this case, the regions of high shear stress, due to the spreading surfactant in the wake, help to prevent the shedding filaments from connecting normally to the free surface. Thus a stretching and tilting occurs and a horseshoe vortex system is created.

To meet the vorticity conditions that both a finite amount of vorticity is observed at the free surface and that a vortex filament cannot terminate within the bulk the vortex filaments below the free surface must shed from the cylinder and pair and 

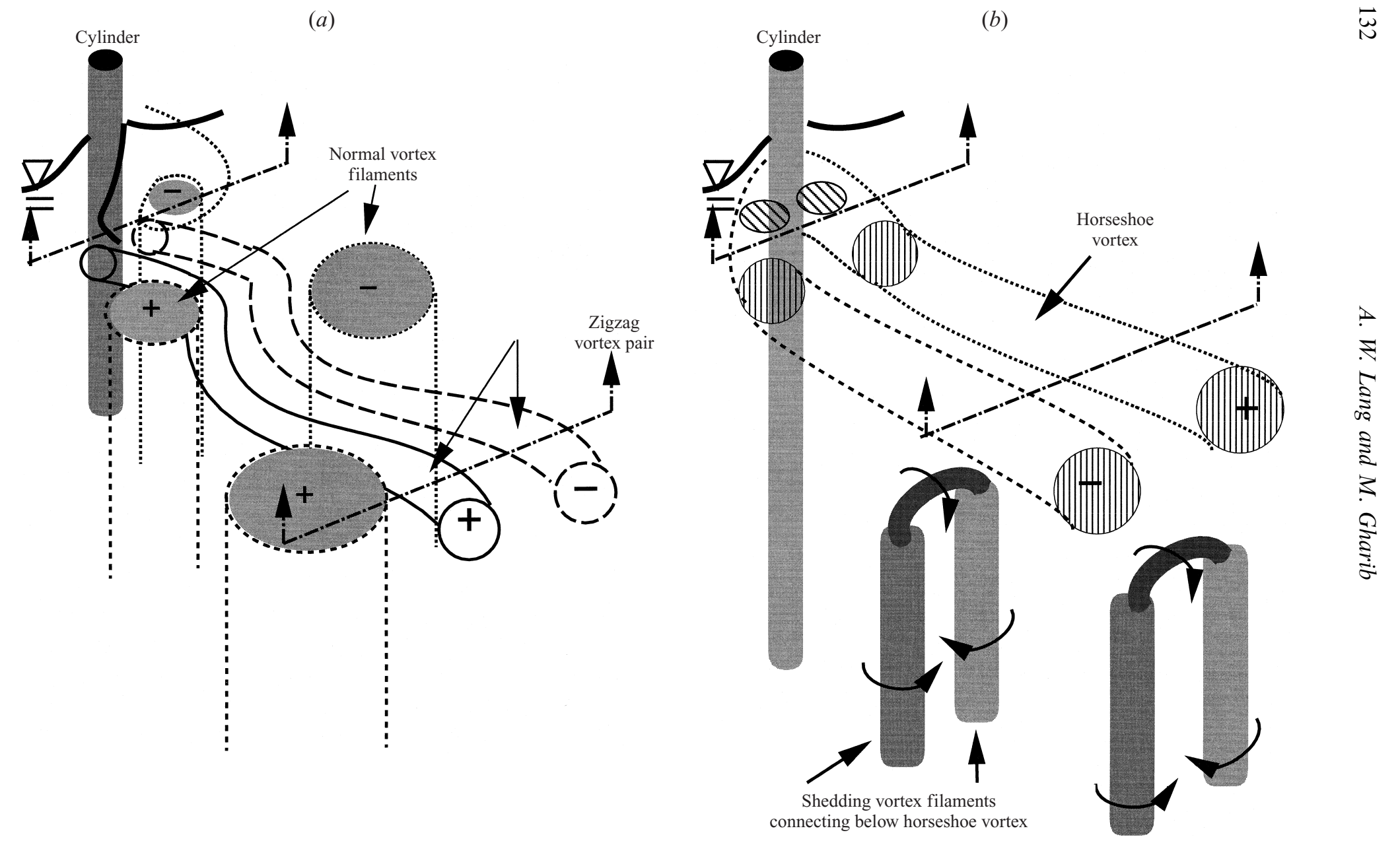

Figure $18(a, b)$. For caption see facing page. 

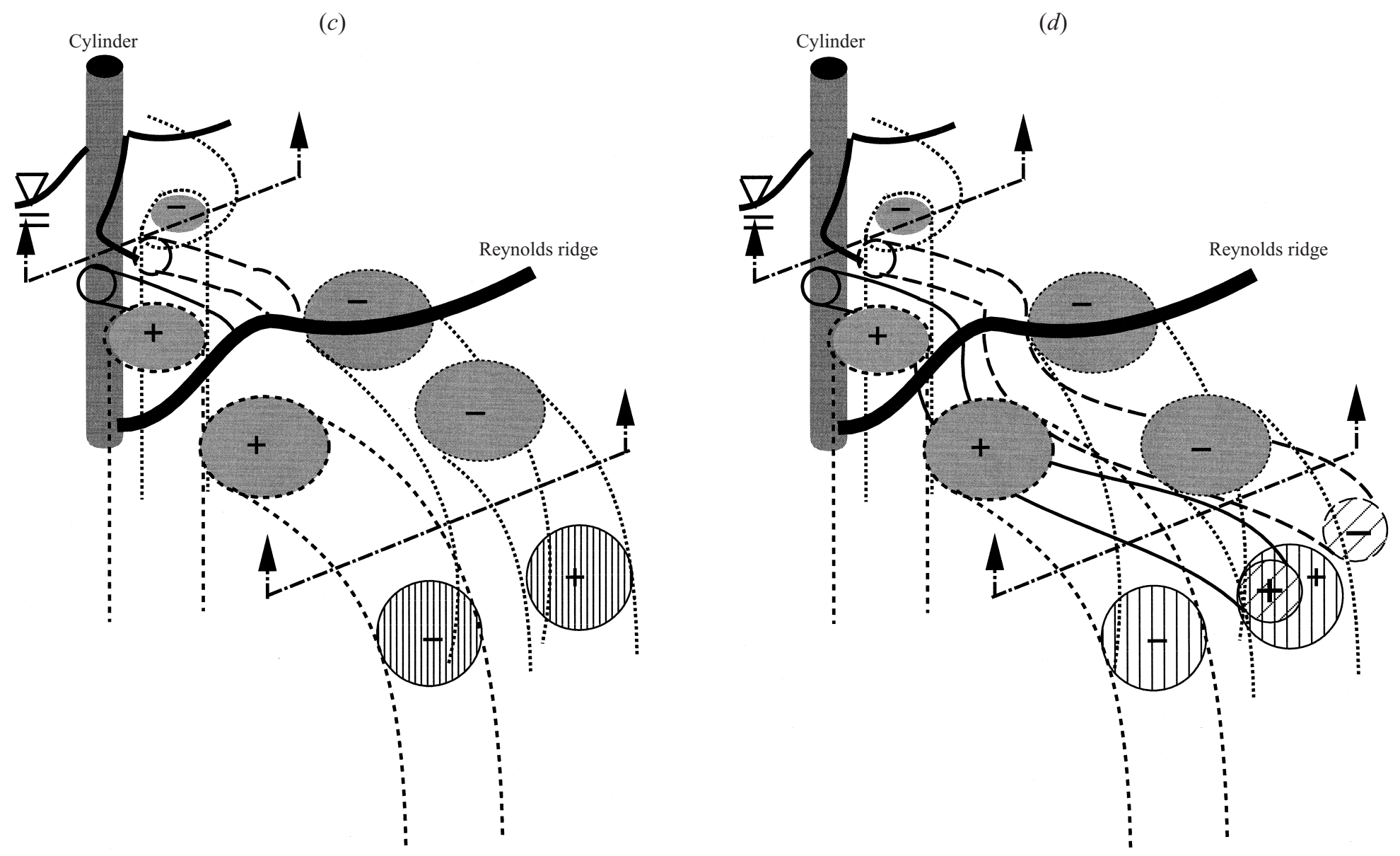

FIGURE 18. Model schematic of the cylinder wake for $(a)$ a clean surface, $(b)$ a contaminated surface, $(c)$ a strong Reynolds ridge in the cylinder wake, and $(d)$ a weak ridge in the cylinder wake. These are lower down in the near region of the free surface, so all flows resemble the von Karman vortex street. 
reconnect below the horseshoe vortex as shown in the model. This pairing is evident in figure $12(b)$ as stated in $\S 4.2$. These connecting filaments joining the opposite-sign vortex tubes on average form the weak negative-vorticity layer below the positivevorticity boundary layer observed for the contaminated surface case (figure 17b). The weakness of this layer is due to the averaging of the filaments as they move from left to right through the cross-section. This must occur, otherwise an approaching infinite amount of vorticity would be connected to the horseshoe vortex from all the filaments that have passed through the wake. It was also observed that further downstream the strength of the horseshoe vortex pair decreased. This is due to the disconnection of the filaments and decay of the convected vorticity that was left behind in the horseshoe vortex after disconnection.

Finally, two models of the flow where the ridge is in the near cylinder wake are shown in figures $18(\mathrm{c})$ and $18(d)$. These flows are a combination of the first two models presented where the strength of the ridge (the surface tension gradient or shear at the surface) is larger for the higher Re case. The initial wake ahead of the ridge corresponds to the clean case; however when the convecting vortex filaments interact with the ridge they get stretched or sheared and tilted at the surface proportionally to the surface tension gradient. In the strong ridge case, a conversion of the flow to the contaminated model is observed with the appearance of one pair of vortex structures at the surface. However, the weaker ridge case still shows evidence of the zigzag pair in the data. Thus the weaker ridge does not stretch the shedding filaments to as large an extent and the non-converted surface-normal vorticity is still large enough to cause significant surface curvature such that the zigzag vortex pair is generated.

\section{Concluding remarks}

The results show that for the case studied here the flow field in the vicinity of the free surface was altered dramatically due to the production of vorticity from surface tension gradients associated with surfactants, as well as by the presence of a boundary layer generated by an upstream Reynolds ridge. The damping effects of surfactants on free-surface deformations is already well known; however in this case it was shown that surfactants altered the flow near the free surface significantly by altering the free-surface boundary condition. In the case of a flat clean surface, it is known that vortex filaments must connect normally at a shear-free surface. However, it was shown that the surface tension gradients may dramatically alter the connection process and provide a mechanism of vorticity conversion from one component to another depending on the 'strength' of the gradient or shear stress disrupting the flow. Also, the various means of vorticity production at a free surface as given by equation (2) (specifically the presence of surfactants and surface curvature) have been accounted for in this flow field.

The relevance of these results to the study of turbulence near a free surface is quite evident. By understanding these lower Reynolds number flow fields, insight is gained into how higher Reynolds number or turbulent flow fields may behave under similar conditions. This deeper understanding will help lead future work in modelling and controlling flows such as those found at ocean surfaces. The results presented show the tendency of large surface shear stresses, associated with the presence of surfactants, to redirect vortex filaments from the normal component to the surfaceparallel component and to generate vorticity in the surface-parallel direction as well. This is evident in the boundary layer that forms beneath a Reynolds ridge.

Finally, one can see the significance of these results with respect to ship wakes. It 
is evident from the discussion earlier that the diverging surface current observed to persist in the ship wake is one reason for the wake to remain visible at the surface for so long, through the formation of a concentrated band of surfactants in which waves are damped. However, this band of surfactants in the wake also increases in concentration due to the upwelling flow at the centre region and bubbles (due to air entrained in the near region of the ship), both of which carry surfactants to the surface. Keeping in mind that the far-field wake of a ship is also a highly turbulent flow field, the importance of understanding the distribution and dissipation of vorticity within this vortex-dominated wake is clearly evident. Thus, if the presence of surfactants has the effect of redirecting the vorticity component near a free surface to the surface-parallel direction, this may have the consequence of 'feeding' vorticity into the large counter-rotating pair and in essence contributing to its long persistence. As a result when studying flows at a free surface, such as those found in ship wakes, and attempting a model of a particular flow, the effects of surfactants on free-surface flows may not be negligible and must be taken into account.

This work has been supported by the Office of Naval Research, ONR-URI grant N00014-94-1-0596 and was performed at GALCIT. The authors would like to thank Professor Thomas Roesgen for his help and insight. In addition, we wish to thank Professor Anthony Leonard for his assistance. The National Science Foundation and Amelia Earhart Fellowship Award also gave financial support to first author Amy Warncke Lang during her graduate studies.

\section{REFERENCES}

Bernal, L. P., Hirsa, A., Kwon, J. T. \& Willmarth, W. W. 1989 On the interaction of vortex rings and pairs with a free surface for varying amounts of surface active agent. Phys. Fluids A $\mathbf{1}$, 2001-2004.

Chang, C. \& Franses, E. 1995 Adsorption dynamics of surfactants at the air/water interface: a critical review of mathematical models, data, and mechanisms. Colloids and Surfaces A 100, $1-45$.

DAviEs, J. T. 1966 The effects of surface films in damping eddies at a free surface of a turbulent liquid. Proc. R. Soc. Lond. A 290, 515-526.

Edwards, D. A., Brenner, H. \& Wasan, D. T. 1991 Interfacial Transport Processes and Rheology. Butterworth-Heinemann.

Gharib, M. \& Weigand, A. 1996 Experimental studies of vortex disconnection and connection at a free surface. J. Fluid Mech. 321, 59-86.

Hirsa, A., HARPER, J. \& KIM, S. 1995 Columnar vortex generation and interaction with a clean or contaminated free surface. Phys. Fluids 7, 2532-2534.

Hirsa, A. \& Willmarth, W. W. 1994 Measurements of vortex pair interaction with a clean or contaminated free surface. J. Fluid Mech. 259, 25-45.

Kenning, D. \& CoOper, M. 1966 Interfacial circulation due to surface-active agents in steady two phase flows. J. Fluid Mech. 24, 293-306.

Lugt, H. J. 1987 Local flow properties at a viscous free surface. Phys. Fluids 30, 3647-3652.

Lyden, J. D., Lyzenga, D. R. \& Shuchman, R. A. 1986 Analysis of synthetic aperture radar imagery of surface ship wakes. Proc. IGARSS '86 Symp., pp. 801-805.

Marmorino, G. O. \& Trump, C. L. 1996 Preliminary side-scan ADCP measurements across a ship's wake. J. Atmos. Ocean. Tech. 13, 507-513.

Meadows, L., Meadows, G., Troesch, A., Cohen, S., Beier, K. P., Root, G., Griffen, O. M. \& SwEAN, T. F. JR 1994 Lagrangian velocity profiles in the wake of a high speed vessel. Ocean Engng 21, 221-242.

Roesgen, T., LANG, A. W. \& Gharib, M. 1998 Fluid surface imaging using microlens arrays. Exps. Fluids 25, 126-132. 
ScotT, J. C. 1982 Flow beneath a stagnant film on water: the Reynolds ridge. J. Fluid Mech. 116, 283-296.

Tryggvason, G., Abdollahi-Alibeik, J., Willmarth, W. \& Hirsa, A. 1992 Collision of a vortex pair with a contaminated free surface. Phys. Fluids A 4, 1215-1228.

TSAI, W. \& YUE, D. 1995 Effects of soluble and insoluble surfactant on laminar interactions of vortical flows with a free surface. J. Fluid Mech. 289, 315-349.

WALKER, D. 1997 On the origin of the 'surface current' in turbulent free-surface flows. J. Fluid Mech. 339, 275-285.

Warncke, A., Gharib, M. \& Roesgen, T. 1996 Flow measurements near a Reynolds ridge. Trans. ASME: J. Fluids Engng 118, 621-624.

Weigand, A. \& Gharib, M. 1997 On the evolution of laminar vortex rings. Exps. Fluids 22, 447-457.

WiLlert, C. E. 1992 The interaction of modulated vortex pairs with a free surface. PhD thesis, University of California, San Diego.

Willert, C. E. \& Gharib, M. 1991 Digital particle image velocimetry. Exps. Fluids 10, 181-193.

Willert, C. E. \& GHARIB, M. 1997 The interaction of spatially modulated vortex pairs with free surfaces. J. Fluid Mech. 345, 227-250. 